
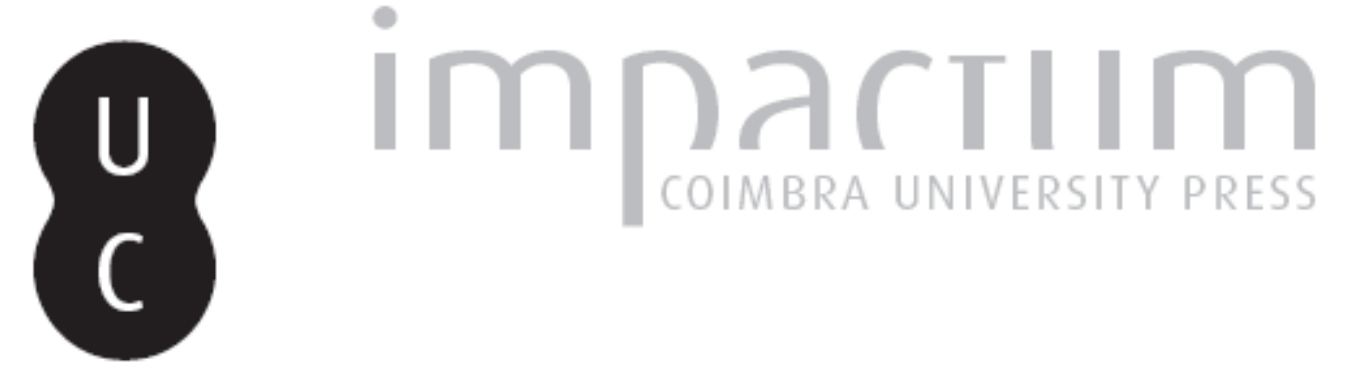

\title{
Art et société d'après les stèles funéraires de Cárquere
}

\author{
Autor(es): Caron, Laurent
}

Publicado por: Imprensa da Universidade de Coimbra

URL persistente:

URI:http://hdl.handle.net/10316.2/45443

DOI:

DOI:https://dx.doi.org/10.14195/1647-8657_35_4

Accessed : $\quad$ 26-Apr-2023 11:58:03

A navegação consulta e descarregamento dos títulos inseridos nas Bibliotecas Digitais UC Digitalis, UC Pombalina e UC Impactum, pressupõem a aceitação plena e sem reservas dos Termos e Condições de Uso destas Bibliotecas Digitais, disponíveis em https://digitalis.uc.pt/pt-pt/termos.

Conforme exposto nos referidos Termos e Condições de Uso, o descarregamento de títulos de acesso restrito requer uma licença válida de autorização devendo o utilizador aceder ao(s) documento(s) a partir de um endereço de IP da instituição detentora da supramencionada licença.

Ao utilizador é apenas permitido o descarregamento para uso pessoal, pelo que o emprego do(s) título(s) descarregado(s) para outro fim, designadamente comercial, carece de autorização do respetivo autor ou editor da obra.

Na medida em que todas as obras da UC Digitalis se encontram protegidas pelo Código do Direito de Autor e Direitos Conexos e demais legislação aplicável, toda a cópia, parcial ou total, deste documento, nos casos em que é legalmente admitida, deverá conter ou fazer-se acompanhar por este aviso.

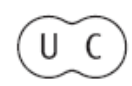


UNIVERSIDADE DE COIMBRA

FACULDADE DE LETRAS

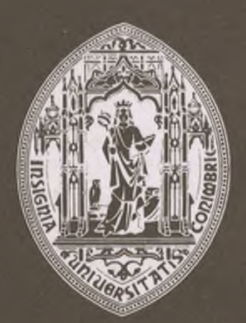

\section{CONIMBRIGA}

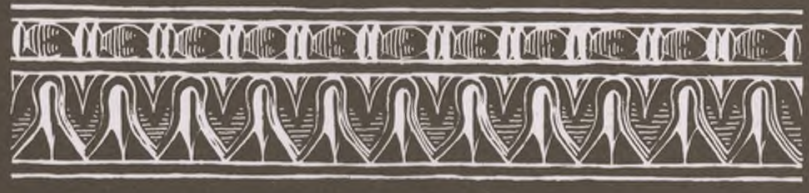

VOLUME XXXV - 1996 


\section{LAURENT CARON}

Arqueólogo. Mestre em Historia Antiga pela Universidade de Bordéus III

\section{ART ET SOCIÉTÉ D'APRÈS LES STÈLES FUNÉRAIRES DE CÁRQUERE} “Conimbriga" XXXV (1996) p. 69-106

RESUMO: Desde o final do séc. XIX que a localidade de Cárquere tem produzido um grande número de estelas funerárias de decoração característica, todas situadas entre o séc. II e o séc. III d. C.. Pela primeira vez, estas estelas são reagrupadas num corpus servindo de base a um estudo sobre a arte funerária e a sociedade de Cárquere. Este estudo demonstra a presença de uma população de origem céltica que, ao mesmo tempo que conservou as suas tradições artísticas e onomásticas, se adaptou à cultura latina. $\mathrm{O}$ artigo que se apresenta foi feito a partir de uma tese de mestrado defendida na Universidade Michel de Montaigne - Bordeaux III.

RESUMÉ: Depuis la fin du XIX ${ }^{\mathrm{e}}$ siècle, le village de Cárquere a livré un très grand nombre de stèles funéraires au décor caractéristique, toutes situées entre le $\mathrm{II}^{\mathrm{e}}$ et le III ${ }^{\mathrm{e}}$ siècle ap. J.-C. Pour la première fois, ces stèles sont regroupées dans un corpus servant de base à une étude sur l'art funéraire et la société de Cárquere. Cette étude a démontré la présence d'une population d'origine celtique conservant ses traditions artistiques et onomastiques, tout en s'adaptant à la culture latine. L'article présenté ici est tiré d'un travail de D. E. A. présenté à l'Université Michel de Montaigne - Bordeaux III. 
(Página deixada propositadamente em branco) 


\section{ART ET SOCIÉTÉ D'APRÈS LES STÈLES FUNÉRAIRES DE CÁRQUERE}

\section{INTRODUCTION GENERALE}

Le Musée National d'Archéologie de Lisbonne abrite dans ses réserves un bon nombre de stèles funéraires venant de toutes les régions $\mathrm{du}$ Portugal. C'est en rassemblant les stèles provenant de Cárquere qu'apparait l'importance que ce village revêt pour l'épigraphie romaine du pays. Une trentaine de monuments a pu être répertoriée pour le moment. En dressant une premiere liste de stèles trouvées à Cárquere et conservées dans divers musées ou ayant seulement laissé une trace dans un article, nous nous apercevons rapidement que Cárquere constitue un noyau épigraphique très important.

Le but de notre travail est de rassembler, pour la première fois, toutes les stèles provenant de Cárquere dans un corpus. Quelques études ont déjà été réalisées dans le passé sur ce sujet mais aucune n'a regroupé l'ensemble des stèles. Ce corpus, une fois terminé servira de base de données pour une première étude sur la société et l'art funéraire de Cárquere. Si l'évolution du comportement de la société indigène lusitanienne face à la domination romaine est de mieux en mieux connue dans le domaine de l'onomastique et des structures familiales, la position de l'art et des rites funéraires, par contre, est un peu plus ignorée. Les stèles de Cárquere vont donc nous permettre de mieux comprendre la société indigène de cette antique localité et de révéler les tendances artistiques de ses habitants. 


\section{INTRODUCTION}

\section{Intérêt de Cárquere}

\section{Localisation}

Cárquere est un petit village appartenant à l'arrondissement (concelho) de Resende et au district (distrito) de Viseu se situant dans la région de $Y$ Alto Douro. A partir de Lamego, il faut parcourir environ trente cinq kilomètres de route montagneuse et sinueuse pour parvenir à la petite ville de Resende. Même si le village appelé Nossa Senhora de Cárquere débute à quelques mètres de Resende, il reste encore quatre kilomètres de montée pour arriver sur le lieu de notre étude ou se trouvent la mairie, l'église et l'ancien monastère de Nossa Senhora de Cárquere.

De ce lieu, le village domine le fleuve Douro. L'intérêt historique de ce village est important car il se situe sur le territoire antique soit des Coilarni, soit des Paesuri, peuples ayant participés à la construction du pont d'Alcantara ('). La frontière de ces deux peuples n'est pas clairement définie. J. Alarcâo voit Cárquere comme capitale des Paesuri et Lamego comme celle des Coilarni sans que toutefois cela ait pu être certifié par des documents littéraires ou épigraphiques. Mais il est vrai que les découvertes archéologiques, toujours fortuites, faites à Cárquere, laissent indiquer la présence d'une occupation humaine assez importante à l'époque romaine.

\section{Les découvertes archéologiques}

Outre sa tour seigneuriale et son monastère, le nom de Cárquere est plus connu dans le milieu archéologique par les découvertes qui y ont été faites. En effet, plus de soixante stèles funéraires ont été trouvées dans ce village.

(') Sur ce sujet voir les recherches de J. AlarCão: $O$ domínio romano em Portugal, Lisboa, 1988; "Identificação das cidades da Lusitânia portuguesa e dos seus territórios", Les villes de Lusitanie romaine. Hierarchie et territoire. Table ronde intemationnale du CNRS, Talence, 8-9 décembre 1988, Collection de la Maison des Pays Ibériques, 42, Paris, 1990, pp. 21-34. 
Les premières découvertes remontent à 1888 ou quelques temps avant. Sept stèles furent publiées par J. Leite de Vasconcellos dans la revue Revista Archeolòica e Histórica $\left(^{2}\right)$. Deux ans plus tard, elles sont de nouveau publiées dans la revue $O$ Archeologo Português avec deux nouvelles inscriptions ( $\left.{ }^{3}\right)$. J. Leite de Vasconcellos confirme l'intérêt archéologique de Cárquere dans son ouvrage Religiões de Lusitânia $\left(^{4}\right)$ par la description de l'ornement des faces latérales de ces monuments funéraires et par la reproduction de deux nouvelles stèles. Enfin, en 1929, il publie deux nouvelles inscriptions provenant de Cárquere dans $O$ Archeologo Português $\left(^{5}\right)$.

Il faut attendre ensuite 1951 pour que Cárquere se distingue avec la découverte et la publication par E. Jalhay de douze nouvelles stèles funéraires trouvées autour du cimetière $\left(^{6}\right)$. En 1956, S. Lambrino parle de 45 stèles de Cárquere abritées au Musée Leite de Vasconcellos, actuel Musée National d'Archéologie ( $\left.{ }^{7}\right)$.

C'est en 1972, lors de la construction d'une route, que deux sépultures sont mises au jour $\left(^{8}\right)$. Il s'agit de deux incinérations en urnes signalées chacune par une stèle funéraire.

Enfin, les dernières découvertes remontent à 1989 lors de l'ouverture d'une nouvelle route $\left({ }^{9}\right)$. Deux fragments de stèles furent recueillis à cent mètres du monastère.

Outre ces trouvailles, les archéologues notèrent la présence de céramiques romaines, fûts et chapiteaux de colonnes, monnaies et

(2) J Leite de Vasconcellos, "Antiguidades de Cárquere", Revista Archeologica e Histórica, II, 1888, pp. 113-115.

(3) J. Leite de VAsconcellos, "Antiguidades de Cárquere”, O Archeòlogo Português, V, 1900, pp. 206-212.

$\left({ }^{4}\right)$ J. Leite de Vasconcellos, Religiões da Lusitânia, III, 1913, p. 414, 440 et pp. $454-456$.

$\left(^{5}\right)$ J. Leite de Vasconcellos, "Epigraphia do Museu Etnológico (Belém). Inscrições romanas”, O Archeòlogo Português, XXVIII, 1929, pp. 209-221.

$\left.{ }^{6}\right)$ E . JALHAY, "Lápides romanas da região de Cárquere, Resende", Brotéria, LI1, 1951, pp. 70-85.

(7) S LAMBRino, "Les inscriptions latines du musée Leite de Vasconcelos", O Archeologo Português, nova série, III, 1956, p. 6.

$\left(^{8}\right)$ V. CoRreia, "Arqueologia de Cárquere", Obras, IV, 1972, pp. 261-263.

( ${ }^{9}$ J. Reigota, "Estela a Valerio Reburro de Cárquere (Resende)", Ficheiro Epigráfico, 32, 1989, n.. 147 et "Fragmento de estela funerária de Cárquere (Resende)”, Ficheiro Epigráfico, 32, 1989, n. ${ }^{\circ} 148$. 
autres objets divers. En face de l'église, devant le cimetière, subsiste encore une colonne entière, replacée à cette endroit.

Nous pouvons donc conclure qu'à chaque fois que des travaux ont remué la terre à Cárquere, que ce soit dans le cimetière ou près de celui-ci et du monastère, des stèles funéraires ou des fragments sont apparus. $\mathrm{Si}$ ces monuments avaient pu être attribués uniquement à la construction de la tour seigneuriale ou du monastère, comme pierre de réemploi, la decouverte de deux sépultures démontrent que nous sommes bien en présence d'un contexte funéraire sur ce lieu.

\section{Etude}

\section{Les données}

Pour cette étude, deux sources ont été exploitées: la bibliographie et les stèles. Ces deux sources sont complémentaires afin d'obtenir les données les plus complètes sur un monument.

\section{La bibliographie}

Le premier article sur les stèles de Cárquere date de 1888 ou J. Leite de Vasconcellos est le premier à s'interresser à ces inscriptions. A partir de là, la bibliographie sur Cárquere va constamment s'enrichir soit par des publications de nouvelles stèles, soit par quelques études sur les inscriptions et l'onomastique $\left({ }^{10}\right)$. Ces articles sont plus ou moins complets. Certains mentionnent seulement des inscriptions ou des stèles, d'autres en donnent le texte et les dimensions avec parfois un dessin. La bibliographie est donc plus ou moins détaillée et riche selon les articles et aussi selon la date de leur publication, ce qui pose souvent des problèmes de dimensions, de lecture et de localisation des stèles. De plus, aucune étude ne se penche sur le fait que Cárquere abrite une

$\left.{ }^{10}\right)$ M.M. A. DIAS, "Inscrições romanas de Cárquere, Resende, na colecção epigráfica do Museu Nacional de Arqueologia e Etnologia", $O$ Archeòlogo Português, série IV, 4, 1986, pp. 185-202; "Antroponimia de Cárquere, Resende, Viseu (Lusitânia Portuguesa)", Actas del IV Coloquio sobre Linguas e Culturas Paleohispanicas (Vitoria/Gasteiz, 6-10 mayo 1989), Veleia, 2-3, 1987, pp. 195-203.

Conimbriga, 35 (1996) 69-106 
grande nécropole funéraire d'époque romaine, seuls semblent compter la stèle et son inscription.

Toutefois, il est bien décevant de s'apercevoir qu'il n'existe aucun corpus des inscriptions de Cárquere ni aucune étude d'ensemble pour un site qui a livré plus d'une soixantaine de stèles, formant ainsi le deuxième noyau épigraphique du pays.

\section{Les stèles}

De Cárquere proviennent actuellement 62 stèles ou fragments, toutes de granite. Ces monuments ont été répartis dans différents musées. Le Musée National d'Archéologie de Lisbonne en abrite trente, les musées de Guimarães et de Lamego en possèdent trois chacun. La Casa do Infante à Porto doit aussi en abriter quelques uns. Mais certaines stèles enregistrées dans la bibliographie restent introuvables. Soit elles ont totalement disparu, soit elles restent stockées dans les réserves de ces musées et deviennent avec le temps non identifiables (c'est le cas pour le musée de Lisbonne) car trop usées ou couvertes de lichens, ce qui rend la lecture très difficile, voire parfois impossible.

L'exploitation de ce type de document a donc été souvent aussi difficile et laborieuse que l'étude de la bibliographie. Il a fallu parfois avoir recours aux publications pour identifier une stèle qui, une fois étudiée, complète les données bibliographiques.

\section{Méthode de travail}

La méthode de travail utilisée pour cette étude est constituée de deux phases: le dépouillement de la bibliographie et l'étude de la stèle sur son lieu de conservation.

\section{Dépouillement de la bibliographie}

La première étape de cette phase a été de dresser une liste de toutes les inscriptions publiées afin d'obtenir une idée sur le nombre de documents et sur le type d'inscription existant. Ensuite, chaque stèle se voit attribuer une fiche où y est indiqué le maximum de renseignements 
qui a pu être obtenu: forme, dimensions, décor et surtout le lieu de conservation du monument et son numéro d'inventaire quand celui-ci en possède un.

\section{Etude des stèles}

Une fois que le maximum d'informations a pu être tiré de la bibliographie, la seconde phase consiste à se déplacer sur le lieu de conservation de la stèle dans le but de s'assurer, premièrement, qu'elle est bien dans le lieu indiqué, puis de l'étudier. Ma présence dans les réserves du Musée National de Lisbonne m'a permis aussi d'enquêter sur un certain nombre de stèles présumées disparues et d'en retrouver une partie grâce à la liste dressée lors de la première étape. Par cette enquête, j'ai pu retrouver quelques stèles provenant de Cárquere et inconnues des publications. Ces stèles inédites ont été étudiées en même temps que les autres.

L'étude des stèles a été la plus complète possible. Toutes les dimensions ont été prises afin de les comparer à celles données dans les articles et aussi pour rajouter la hauteur des lettres d'un texte dans bon nombre de cas. Une lecture personnelle est primordiale pour comparer et corriger les différentes interprétations d'une inscription. Les particularités du monument et son état sont aussi enregistrés et, quand le temps est disponible, un décalque de la stèle est réalisé sur une surface plastique transparente. Enfin des photos sont prises quand cela est autorisé afin d'avoir un support visuel lors de la mise en fiche du monument.

\section{Présentation des fiches}

Dans cet article, les inscriptions funéraires sont regroupées dans l'annexe II et présentées le plus simplement possible (lieu de découverte, texte, traduction et bibliographie). Dans le document originali ${ }^{11}$ ), chaque inscription possède une fiche ayant pour modèle la fiche PETRAE du

(n) Deux exemplaires de la thèse de D. E. A. se trouvent à la bibliothèque de la Maison de l'Archéologie, Université de Bordeaux III, Talence (France), et un a été remis à l'Institut Archéologique de Coimbra (Portugal). 
Centre Pierre Paris de Talence. Cette fiche s'organise en trois parties: un descriptif du support, un descriptif du texte et les observations.

Le descriptif du support réunit toutes les informations obtenues sur l'état de la stèle, sa forme, ses décors, ses dimensions (longueur/ /largeur/épaisseur), sa localisation ainsi que le numéro d'inventaire. Puis, viennent les renseignements concernant l'inscription. Le champ épigraphique et son état de conservation sont détaillés. Parfois, ces données sont manquantes quand la stèle a disparu et qu'aucune photo n'existe dans la bibliographie. Un dessin du monument est peu fiable pour ce genre de renseignement. Le style d'écriture est ensuite décrit et, quand cela est possible, soit donné dans la bibliographie, soit étudié sur place, la hauteur des lettres est indiquée. Avant de retranscrire le texte, toute la bibliographie connue sur la stèle est réunie.

Le texte est d'abord retranscrit en capitale tel qu'il a été lu puis développé ensuite. Quand plusieurs auteurs ont publié la même inscription, les variations de lectures sont indiquées. Après cela vient la ou les traduction(s) selon la nature du texte. En effet, certains textes offrent plusieurs interprétations de lecture.

La dernière partie regroupe toutes les observations qui peuvent être faites soit sur le support, soit sur le texte. Ainsi, il est plus facile de regrouper des types de stèles ou de décors et d'observer les particularités de l'inscription et des noms.

Certaines fiches apparaissent ainsi complètes grace à certaines publications bien détaillées ou à une étude sur place. D'autres possèdent très peu de renseignements du fait que la stèle ait disparu en laissant soit un dessin et quelques dimensions dans un article, soit une description sommaire accompagnée ou non d'une photo.

\section{Problématique}

Ce sujet sur 1'“Art et la société d'après les stèles funéraires de Cárquere "a été choisi car trois centres d'intérêt se dégagent.

Premièrement, il s'agit de regrouper toutes les stèles de Cárquere sous forme de fiches afin de constituer la base d'une étude. $\mathrm{Vu}$ la quantité de stèles retrouvée et le nombre d'articles qui y ont été consacrés, Cárquere mérite une plus grande attention et au moins un corpus d'inscriptions.

Ce corpus d'inscriptions nous permettra de dresser la liste des noms qui apparaissent dans les textes et d'étudier une partie de la 
société de Cárquere. Ainsi, nous pourrons observer l'attitude de l'onomastique indigène face à la pénétration de l'onomastique latine et tenter de distinguer les structures familiales présentes.

Grace à ces fiches et à la description des monuments, une étude du travail et du décor des stèles peut être commencée. En observant si il existe un ou plusieurs types de stèles et si le décor est propre ou non à Cárquere nous pourrons nous poser la question de l'existence d'un atelier de graveur à Cárquere.

\section{ART ET SOCIÉTÉ}

\section{Art et symbolisme sur les stèles}

Plus de la moitié des stèles de Cárquere sont décorées. L'étude des décors et de la forme des stèles nous renseignera sur les préférences artistiques de la population dans le domaine funéraire et sur la possibilité de la présence d'un atelier de graveur à Cárquere.

\section{Les types de stèles}

Le type le plus répandu, à Cárquere, est celui de la stèle à fronton triangulaire: $35 \%$ des monuments. Généralement, il s'agit d'un fronton simple, parfois bordé d'une moulure. Plusieurs groupes peuvent être définis à l'intérieur de ce type. On trouve, premièrement, des stèles rectangulaires, peu hautes, surmontées d'un fronton aux pentes douces (n. $\left.{ }^{\circ} 3,32,40\right)$. Le second groupe est composé de stèles très hautes, au fronton plus pentu $\left(\mathrm{n} .^{\circ} 6,17,21,27,45,46,50,51\right)$. Souvent, deux volutes ornent la base du fronton. Le tympan est souvent décoré, soit par des motifs astraux, soit par une niche abritant une figurine en relief. L'origine de ce type de stèle vient du modèle du temple romain. Cette influence romaine est plus marquante sur une stèle de Bracara Augusta où le fonton est soutenu par deux colonnettes en relief, représentant ainsi l'image du temple ${ }^{12}$ ). La présence de ce type de forme ne signifie

(12) J. L. VASCONCELLOS, Religiões da Lusitânia, vol III, Lisboa, 1913, p. 414; C. $L L / /, 2425$.

Conimbriga, 35 (1996) 69-106 
pour autant une adoption de la religion romaine de la part du défunt mais représente plutôt d'une préférence de ce type de stèle.

Le second type est celui des stèles antropomorphes ou discoidales. Ce type est peu représenté (6,3\%) mais témoigne d'une survivance des sources indigènes primitives. Ces stèles sont surmontées d'un disque ou un arc outrepassé contenant soit un visage, soit une rosace (n. $\left.{ }^{\circ} 7,8,54\right)$. Parfois, deux excroissances arrondies symbolisent les bras $\left(n .^{\circ} 8\right)$. Ce genre de figuration, très répandu dans la Péninsule Ibérique $\left({ }^{13}\right)$, dérive de la stylisation de la figure humaine.

Un troisième ensemble regroupe toutes les stèles n'appartenant pas à ces deux types. La plupart de ces stèles sont cassées dans leur partie supérieure. Leur forme entière est ainsi inconnue. D'autres, sont des monuments rectangulaires (n. $\left.{ }^{\circ} 23\right)$ ou élipsoïdaux ( $\left.^{\circ} 16\right)$ divisés en deux parties: une base grossièrement taillée s'enfouissant dans la terre, surmontée d'une surface mieux travaillée servant de support à l'inscription funéraire.

L'influence romaine est donc prédominante dans le choix des formes des monuments funéraires avec l'adoption de la stèle à fronton mais cette influence semble se limiter à la forme, le décor, quant à lui, semble s'inspirer d'autres sources.

\section{Les types de décors}

La décoration des stèles de Cárquere est peu variée. Trois thèmes seulement peuvent être dissociés: les symboles astraux, les figures en relief dans des niches et la décoration des faces latérales par des palmes stylisées ou des tridents (Annexe I, Tableau I).

\section{Les symboles astraux}

Les symboles solaires sont beaucoup employés sur les stèles sous la forme de rosaces, roues ou disques gravés ou sculptés $(25,4 \%$ des stèles possèdent un tel symbole). La rosace est presque toujours tracée

(13) E. FrankowsKI, "Estelas discoideas de la Peninsula Iberica, junta para amplicacion de estudios et investigaciones sientificas.", Memoria, 25, 1920, p. 39, fig. 8, p. 151, fig. 66 .

Conimbriga, 35 (1996) 69-106 
au compas et sculptée en bas relief (An. I, tab. II/a). Les six pétales sont géométriquement parfaits. Les roues et les cercles garnis sont généralement gravés et ornés d'un point en leur centre ou de rayons pour les premiers (An. I, tab. II/b) et de lignes en contre courbe ou de "svastika flamboyante" pour les seconds (An. I, tab. Il/c et d). Les grands disques des stèles antropomorphes contiennent soit une figure humaine soit une rosace $\left(n .^{\circ} 7\right)$. Ces symboles, d'origine indigène se rencontrent en Gaule et dans la Péninsule Ibérique (14). En Gaule, ils ornent presque exclusivement les stèles indigènes $\left({ }^{15}\right)$. Au Portugal, ces signes solaires sur des stèles d'époque romaine proviennent directement des motifs décoratifs de l'architecture et de l'orfèvrerie proto-historique du Nord. Ces mêmes décors se retrouvent sur des bijoux et des plaques décoratives des monuments du Castro de Citânia de Briteiros $\left({ }^{16}\right)$. Les celtes voyaient dans la mort un passage à une autre vie et croyaient que les défunts s'en allaient au séjour des dieux $\left({ }^{17}\right)$. Le symbole solaire représenterait alors le passage astral vers ce nouveau monde.

Parallèlement à l'emploi des signes solaires, se développe l'utilisation du croissant lunaire, présent sur $8 \%$ des stèles (An. I, Tab. II/e). Ce symbole, d'origine orientale, a pénétré dans le monde occidental avec les milieux mystiques qui apparaissent dès le début du III ${ }^{\mathrm{e}}$ siècle ap. J.-C comme les cultes de Cybèle, Mithra et Isis (18). Il a ensuite été adopté dans les milieux indigènes qui se trouvaient en rapport avec les milieux mystiques dans les grandes villes. Cela ne signifie pour autant que les indigènes ont adopté ces nouvelles croyances religieuses. Le croissant lunaire a pu devenir un nouveau moyen de décoration pour exprimer leurs idées sur la mort et la vie après la mort. Le voyage des âmes dans les astres se ferait alors sous une forme différente. Même s'il n'y a pas eu une analogie de croyance, nous pouvons constater qu'il existe une convergence de deux symbolismes, l'un local, l'autre venu de l'orient.

(14) J. J. HATT, La tombe Gallo-romaine, Paris, 1986, p. 334-335.

(15) J J. HATT, p. 387.

(16) Ces plaques sont actuellement visibles au musée "da Sociedade Martins Sarmento" à Guimarães.

(,7) H. Graillot, Le culte de Cybèle, Mère de dieux, à Rome et dans l'empire romain, BEFAR, 107, Paris, 1912, p.264.

(18) J. J. HATT, p. 387.

Conimbriga, 35 (1996) 69-106 


\section{Niches et figurines}

La représentation humaine sur les stèles de Cárquere ne se fait jamais par le moyen de la grande sculpture comme la réalisation en bas-relief de portraits, bustes ou figures sur pied, mais toujours par de petites figurines grossièrement sculptées ou gravées. Ces petits personnages, seuls ou en couple, symbolisent le défunt. Ils sont représentés sur pied avec pour seuls détails leurs principales parties anatomiques (jambes, tronc, bras et tête). Généralement, les traits du visage sont absents soit par omission, soit dus à l'usure du granite.

L'emploi de la figurine en bas-relief va de paire avec celui de la niche. Sur sept stèles comportant un personnage $(11 \%)$, six ont aussi une niche $(9,5 \%)$. Elle est toujours semi-circulaire et est souvent surmontée d'un fronton, rappelant ainsi les petits oratoirs des carrefours et la petite chapelle ou était nichée la divinité. Sur certaines stèles, la niche est surmontée d'un symbole astral. Cette organisation marquerait-elle le souhait d'accéder au monde des dieux de la part du défunt ou serait-elle l'indication d'un fait accompli: "Il est mort et se trouve au royaume des dieux"?. En outre, la présence de deux figurines dans une niche représente-t-elle deux défunts?. Deux cas se présentent. La stèle $n .^{\circ} 21$ possède une niche à deux personnages alors que l'inscription ne signale qu'un défunt: Mia Tongeta. La stèle n. ${ }^{\circ} 52$ a aussi deux figurines dans une niche mais le texte, divisé en deux par une ligne verticale, pourrait différencier deux défunts. Malheureusement, la stèle reste introuvable et le texte donné par J. L. Vasconcellos est incomplet. Ce type de sépulture, appelé bisonum à l'époque chrétienne, n'est donc pas certifié à Cárquere.

\section{Les palmes stylisées et les tridents}

La palme stylisée associée ou non au trident est le motif le plus utilisé sur les stèles. Ce décor est toujours situé sur les côtés latéraux du corps du monument et se répète parfois sur les côtés du fronton. Plus d'un tiers des stèles $(35 \%)$ possèdent cette décoration latérale. Le symbole de la palme unique ou se terminant en trident est le plus représenté $(28,6 \%)$, alors que seulement 4 stèles portent le trident seul $(6,4 \%)$. Cinq types de décor latéral ont pu être répertoriés (An. I, tab. III). Chaque type possède deux variantes. Une stèle peut contenir plusieurs 
types car le décor sur une face latérale n'est pas obligatoirement le même sur l'autre. La palme stylisée à une tige se terminant par un trident est la plus usitée (type III a), suivie de très près par la palme à une tige sans trident (type $\mathrm{V}$ a). La palme appartient au symbolisme végétal toujours présent dans le culte des morts. Chez les romains, elle est le symbole de la victoire sur la mort. Les indigènes, eux, "assimilent la vie latente et mystérieuse des végétaux à la survie de l'âme” (19).

Le professeur J. d'Encamaçâo voit plutôt $\left({ }^{20}\right)$, dans ces décors latéraux, le symbole de la feuille d'if (taxus baccata), arbre totem des Astures. Ces derniers extrayaient des bourgeons de cette plante un puissant poison qu'ils ingéraient collectivement afin d'échapper, par la mort, à l'esclavage des romains vainqueurs. La culture castréjane du Nord-Ouest de la Péninsule Ibérique ayant largement débordé le Douro vers le $\operatorname{Sud}\left({ }^{21}\right)$, il est fort probable que la population de Cárquere connaissait les pouvoirs de cette plante et ait utilisé cette dernière dans leur symbolisme funéraire.

La présence du trident sur les stèles de Cárquere, par contre, est inexplicable. Une partie des palmes stylisées se termine soit par un trident, soit par une fourche à deux dents. Quatre stèles, seulement, sont décorées par un simple trident. Aucun texte ne fait référence au gladiateur retiarius qui utilise cette arme. Cette explication est donc à rejetter. Cette représentation symboliserait-elle tout simplement la fourche, l'outil agricole des défunts?

Il semble certain que la palme stylisée, se terminant par un trident ou non et gravée sur les côtés latéraux de la stèle, est un décor typique de Cárquere. Le musée du Carmo de Lisbonne possède bien une stèle ou est gravée une palme ou un rameau stylisé, mais celui-ci se trouve sur la face principale et il est surmonté d'une représentation d'un vase retourné $\left({ }^{22}\right)$. Une stèle provenant de Meijinhos $\left(\mathrm{n}^{\circ}{ }^{\circ} 15\right)$ et conservé au musée de Lamego porte aussi sur les côtés latéraux un trident gravé (type II a). La décoration et la forme de cette stèle sont les mêmes que

(,9) J. J. HATT, p. 403.

(20) J d'ENCARNAÇão, "O monumento epigráfico, fonte para o estudo das migrações na Península Ibérica".

(21) A. C. F. Silva, A cultura castreja no noroeste de Portugal, Paços de Ferreira, 1986.

(22) J. L. VASCONCELLOS, Religiões da Lusitânia, vol III, Lisboa, 1913, p. 420, fig. 199. 
celles que l'on rencontre sur les monuments de Cárquere. J. L. Vasconcellos avait déjà remarqué, en 1913, que ce décor particulier distinguait les stèles de Cárquere.

\section{La chronologie}

Etablir une chronologie des inscriptions de Cárquere n'est pas chose aisée. En raison de l'absence du contexte funéraire qui aurait pu dater chaque monument par une étude céramologique ou numismatique, nous devons tenter de définir une chronologie à partir de l'anthroponymie de Cárquere. Nous ne reprendrons pas en détail ce travail, déjà effectué par M. M. A. Dias ( $\left.{ }^{23}\right)$, qui place les stèles de Cárquere entre le milieu du Ile siècle ap. J.-C. et le lile siècle ap. J. -C.

Certain textes peuvent être ainsi datés du milieu ou de la fin du II ${ }^{\mathrm{e}}$ siècle ap. J.-C. par le module rectangulaire allongé de leur lettres (n. ${ }^{\circ} 18,35,36,37 \ldots$ ), d'autres par la présence d'un gentilice. Le gentilice Ulpius (n. $\left.{ }^{\circ} 44\right)$ semble être associé à la réorganisation militaire de l'Hispanie au II ${ }^{\mathrm{e}}$ siècle ap. J.-C. ${ }^{(24)}$. Celui d'Aurelius (n. ${ }^{\circ} 11$ ) provient de l'extention de la citoyenneté à tous les habitant de l'Empire par Caracala en $212\left({ }^{25}\right)$. Selon M. M. A. Dias, l'élément le plus tardif vient du nom Afinatus, datable paléographiquement de milieu du lile siècle ap. J.-C. $\left({ }^{26}\right)$.

Les stèles de Cárquere fournissent donc un ensemble stylistique et chronologique homogène et attestent la présence d'une nécropole utilisée tout au long des II $^{\mathrm{e}}$ et le III $^{\mathrm{e}}$ siècles ap. J.-C. Cette homogénéité nous permet de nous interroger sur la possibilité du fonctionnement d'un atelier de graveur à Cárquere.

\section{Un atelier de graveur à Cárquere?}

Le décor des stèles de Cárquere est donc assez restreint. Il se limite à la représentation de symboles astraux, de figurines en relief

(23) M. M. DIAS, "Antroponimia de Cárquere, Resende, Viseu (Lusitânia Portuguesa)”, Veleia, 1987, pp. 195-203.

(24) Ibid, p. 201.

(25) Ibid., p. 201.

(26) M. M. DiAs, "Inscrições romanas inéditas de Cárquere”, p. 192. 
dans des niches et de palmes stylisées et tridents sur les côtés latéraux. La pauvreté stylistique est plus qu'apparente. Elle dénonce une maîtrise stylistique très moyenne, témoignage d'un art local emprunt d'influence étrangère. Cette appellation "produit local" est renforcée par le décor typique des côtés latéraux des stèles ne se rencontrant qu'à Cárquere. Toutes ces données nous permettent d'ébaucher l'hypothèse de la présence d'un atelier de graveur à Cárquere ou dans ses environs. Le monastère et le cimetière recouvrant sûrement la nécropole romaine, il faudrait tenter de localiser la localité antique abritant cet atelier aux alentours de Santa Maria de Cárquere. Les hauteurs du mont sur lequel s'accroche le village pourraient avoir abrité un castro qui aurait survécu à l'époque romaine, mais, à l'exception de la découverte de céramiques, de fûts de colonne et de petits objets proche ou danbs le village, rien ne le certifie actuellement.

\section{La société de Cárquere d'après les inscriptions}

Cette étude est basé sur 35 inscriptions (55,5\%) contenant une référence onomastique utilisable. Les 28 autres inscriptions sont, soit des fragments de stèles où le texte restant est inutilisable, soit des inscriptions complètes ou incomplètes posant des problèmes épigraphiques. Soixante-dix-sept noms ont été répertoriés dont trente-six noms de défunts. L'étude de ces textes va nous permettre d'observer le comportement de l'onomastique latine et de suivre les étapes de sa latinisation.

\section{Quelques problèmes épigraphiques}

Quelques inscriptions de Cárquere ont posé des problèmes épigraphiques en dehors des difficultés de lecture dues à l'usure des monuments. Le premier problème rencontré est celui de l'authenticité du texte. Nous le rencontrons quand l'inscription a disparu et qu'il ne nbous en reste qu'une trace bibliographique. Le texte retransmis peut s'avérer parfois incomplet (insc. n. ${ }^{\circ}$ 52) ou sa lecture erronée? (insc. 26).

D'autres textes de Cárquere posent des problèmes de retranscription. Certains mots possédant une abréviation en une 
ou deux lettres, comme l'inscription n. ${ }^{\circ} 50$, ne peuvent pas être développés avec certitude. La série des inscriptions $\mathrm{n} .^{\circ} 2,8,9$ pose cette dificulté. Dans ces derniers textes, nous ensemble de lettres: F A M A . Cet ensemble peut être accompagné d'autres lettres ou de puncti: F A MA (n. $\left.{ }^{\circ} 8\right)$, A F AM. A (n. $\left.{ }^{\circ} 2\right)$, F A M (n. ${ }^{\circ}$ 9) Faut-il donc se baser sur l'inscription n. ${ }^{\circ} 7$, consacré à $F$. Amoena, pour développer ces trois textes?. Les stèles n. ${ }^{\circ} 8$ et 9 seraient ainsi consacrées à deux $F$. Amoena et la stèle n. ${ }^{\circ} 2$ à A/a(?) Amoena.

En dehors de ces quelques problèmes les épitaphes de Cárquere sont assez stéréotypées. Elles ne fournissent que le minimum de renseignements sur le défunt, mais nous donnent de nombreuses données permettant d'ébaucher une première étude de l'onomastique et de la société de Cárquere.

La pénétration de Fonomastique romaine et la survivance de l'onomastique indigène

La pénétration de Vonomastique romaine

Sur les 35 textes étudiés, $62,9 \%$ contiennent au moins un nomen ou un cognomen latin et $37,1 \%$ conservent un nom indigène sans la présence de l'onomastique latine. Parmi ces noms latins, on trouve quelques gentilices comme.

\section{Aureliuus'A $U$ l p i $u$ s: 1 \\ lulius'3 \\ Valerius: 3}

\section{Iunius:2}

Ces gentilices semblent perdre leur valeur de nom de famile (gens) et sont plus utilisés comme praenomen sous sa forme d'abréviation. A côté des gentilices, on trouve les cognomina, utilisés parfois comme gentilice par les indigènes. La liste des cognomina de Cárquere est la suivante:

Amoena: 3

Avitus: 1

Carus: 1

Clemens: 1 
Fronto: 1

Gracilus: 1

Maelia: 2

Oculatus: 1

Rufinus: 3

Rufus: 7

Sabinus: 1

Saturninus: 1

Severus: 1

Comme nous pouvons le constater le cognomen Æw/ws et son dérivé Rufinus ont eut plus de succès auprès de la population de Cárquere qui semble avoir adopté l'onomastique latine, même si les noms indigènes restent largement utilisés.

maintien de Vonomastique indigène

Sur ces 77 noms enregistrés, 48\% sont des noms indigènes, soit 37 noms répartis en 27 types:

$\begin{array}{ll}\text { Afinatus } & \text { Occarius } \\ \text { Albominus } & \text { Peda } \\ \text { Albura } & \text { Pentilius (2) } \\ \text { Alluqius (2) } & \text { Pintamus } \\ \text { Caino } & \text { Pissirus, Pissirea } \\ \text { Calaetus } & \text { Pon(us?) } \\ \text { Camalus } & \text { Promotus } \\ \text { Casabus } & \text { Reburrus (3) } \\ \text { Cellea } & \text { Sunua }(2) \\ \text { Celtus } & \text { Tancinus } \\ \text { Cumelius (2) } & \text { Tolia. } \\ \text { Ibdoena. } & \text { Tongeta (2), Toceta (1). } \\ \text { Malgeinus. } & \text { Viriatis (2). } \\ \text { Mebdus. } & \end{array}$

En ne considérant que les noms des défunts, 50\% des noms sont indigènes (18) et 33,3\% sont romains (12). Les 16,7\% restant (6) sont des noms indigènes associés à un nom latin, généralement un gentilice:

Iulia Sunua Valerius Reburrus: 2. 


\section{Iulia Tongeta M. Valerius Reburrus \\ Maelia Tongeta}

Nous observons donc un fort maintien de l'onomastique indigène, la moitié des défunts portent un nom indigène et presque la moitié des noms de Cárquere sont indigènes, devant une pénétration importante de l'onomastique latine: $50 \%$ des noms sont latins ou mixtes et dans 33,3\% des cas l'onomastique latine a totalement effacé l'onomastique locale.

Cette survivance du nom indigène s'explique par le système de la filiation indigène. Lorsque l'on est en présence d'un nom indigène et que la filiation est indiquée, dans $70 \%$ des cas le père a aussi un nom indigène. Quand le père a un nom indigène ( $53 \%$ des cas), dans $77,8 \%$ des cas le fils a un nom indigène et dans $22,8 \%$ un nom latin. Quand le père porte un nom latin (47\%), dans la moitié des cas le nom du fils est indigène.

Il existe donc une forte tradition de transmission du nom indigène dans les familles qui est conservée à Cárquere permettant le maintien de l'onomastique locale.

\section{Les étapes de la latinisation de Vonomastique indigène}

Il est préférable de parler de latinisation de l'onomastique indigène plutôt que de romanisation à Cárquere car le système romain n'est jamais utilisé par la population locale lors des trois phases de latinisation qui ont été dégagées.

La première phase n'est définie que par la simple latinisation du nom indigène: Promutus, Afmatus, Pentilius, Cellea, Albura; qui évoluera ensuite vers l'association du nom indigène et du nom latin: Valerius Reburrus, Iulia Sunua, Iulia Tongeta; pour finir par l'emploi uniquement de l'onomastique latine: Ulpius Sabinus, Avitus Gracilis, Valeria Amoena.

Les deux autres phases passent par l'indication de la filiation dans les épitaphes. Seulement 17 textes indiquent une filiation. La seconde phase est représentée par le système de la filiation indigène ou est indiqué le nom du défunt suivi du patronyme au génitif. Même si parfois le nom est latin le système reste indigène. Seulement quatre textes sur les 17 indiquant la filiation retransmettent ce système, soit 23,5\%:

\section{Rufinus Calaeti \\ Avitus Frontonis \\ Ponus/ius Iuni \\ Tongeta Rufi}


L'introduction de la filiation par la lettre $\mathrm{F}$ représente la dernière phase. Ce système est utilisé dans $76,5 \%$ des cas. Il est divisé en trois étapes chronologiques.

La filiation avec uniquement l'onomastique indigène constitue 41,2\% des cas (7 inscriptions). L'emploi de la filiation latine par la lettre $\mathrm{F}$ est le premier indice de la transformation du système indigène: Albonius Cumeli F, Pissirus Mebdi F, Pissirea Tancini F, Sunua Casabi F

L'intégration de l'onomastique latine soit chez le défunt, soit chez le père est le deuxième signe de la transformation du système indigène.Elle est enregistrée trois fois, soit $17,6 \%$ des cas: Viriatis Severi F, Tolia Peda Cari F, Iunius Pintami F lunius Fintami F. Nous pouvons penser que parallèlement à cette évolution se développe l'emploi de l'association du nom latin et du nom indigène.

La dernière étape de ce changement de système est marquée par l'abandon de l'onomastique indigène remplacé par l'onomastique latine. Cet abandon est remarqué trois fois (17,6\%): Rufus Rufi F Rufinus Rufi F, Severus Oculati F

L'évolution entre la seconde phase et la troisième se fait par l'assimilation du système de filiation romain tout en conservant les structures indigènes. La pénétration de l'onomastique romaine accentue ce phénomène de latinisation de l'onomastique indigène aboutissant à l'utilisation unique de l'onomastique romaine. Même si l'on arrive à l'abandon de l'onomastique et du système de filiation indigène, il n'existe, pour le moment, à Cárquere, aucune trace d'utilisation du système romain basé sur le prénom du père en abrégé suivi de $\mathrm{F}$, que l'on trouve dans d'autres régions $\left({ }^{27}\right)$.

Toutefois, nous observons une forte conservation des traditions onomastiques locales par la transmission du nom indigène dans 41,2\% des cas et quand le père a un nom romain, deux fois sur trois il donne au fils un nom indigène. Nous observons dons une indéniable latinisation de l'onomastique indigène tout en conservant un maintien de l'onomastique locale, preuve non pas d'une résistance à la culture latine mais d'une assimilation d'une culture à l'autre $\left({ }^{28}\right)$.

(27) A. Tranoy, La Galice romaine, pp. 368-369.

${ }^{(28)}$ R. ETIENNE et alii, "Assimilation et résistance à la culture gréco-romaine dans le monde ancien", Les dimensions sociales de la romanisation dans la Péninsule Ibérique des origines à la fin de VEmpire (Travaux $\mathrm{du} \mathrm{VI}^{\mathrm{e}}$ Congrès International d'Etudes Classiques - Madrid, septembre 1974), Bucuresti-Paris, 1976, p. 106. 


\section{Les formules rituelles}

La présence des formules rituelles dans les textes constitue aussi un indice de romanisation des habitudes funéraires des indigènes. Sur 63 stèles, 74,6\% possèdent une formule rituelle (47 textes). Parmis les 16 inscriptions restantes, 14 sont incomplètes car le bas de la stèle est cassé. Il n'est donc pas certain que ces 14 textes n'en comportent pas. Seulement deux textes entiers n'ont pas de formules rituelles, ce qui représente $3 \%$ des cas seulement.

Les formules rituelles présentes sur les inscriptions de Cárquere sont peu diversifiées. On retrouve surtout l'invocation aux dieux Manes: D M S, les formules hic situs est et sit tibi terra levis, dont l'abréviation est généralement $\mathrm{H} \mathrm{S} \mathrm{E} \mathrm{e} \mathrm{t} \mathrm{S} \mathrm{T} \mathrm{T} \mathrm{L} \mathrm{e} \mathrm{t} \mathrm{l'indication}$ faciendum curavit ou curaverunt représenté par les lettres $\mathrm{F} C$ précédant le nom ou la catégorie familiale de la personne ayant fait faire la stèle. Dans un texte, la formule $\mathrm{H} \mathrm{S}$ E est remplacée par $\mathrm{H} \mathrm{C}$ : hic cubat ou hic crematus (est) (n. $\left.{ }^{\circ} 35\right)$.

L'invocation D M S est présente sur 41,3\% des stèles. C'est la formule la plus usitée à Cárquere. Sur les 35 textes utilisables, elle n'apparait que 11 fois $(34,3 \%)$. L'invocation consacrée aux dieux Manes n'est jamais utilisée dans les textes indiquant la filiation d'un individu par le système latin: nom du père au génitif suivi de $\mathrm{F}$.

La formule $\mathrm{H} \mathrm{S} \mathrm{E} \mathrm{est} \mathrm{moins} \mathrm{utilisée:} 22,2 \%$ des textes la possèdent et elle est représentée sur 34,3\% des textes étudiés (12 sur 35). Cette formule est plus souvent employée avec le système de la filiation latine.

La formule S T T L est aussi peu utilisée (23,8\%). Elle est présente sur 25,7 \% des inscriptions (9 sur 35). Elle accompagne presque toujours la formule $\mathrm{H} \mathrm{S}$ E et est aussi un peu plus utilisée avec la filiation latine: $38,5 \%$ des textes avec ce type de filiation ont les formules H S E S T T L.

L'indication F C est représentée dans $40 \%$ des textes utilisés mais ne se trouve que sur $28,6 \%$ des stèles. On l'observe plus souvent dans les textes sans filiation (64,3\%), très rarement avec HSE (une fois) et STTL (deux fois). Lorsqu'il est employé dans les textes avec la filiation latine, il est toujours accompagné de HSE et STTL.

Deux groupes se forment donc. Le premier, représenté par les textes sans filiation ou avec la filiation indigène, utilise beaucoup les formules DMS et FC et peu HSE et STTL. Les deux premières formules semblent avoir été introduites en premier dans les épitaphes de Cárquere. Elles 
sont, en tous cas, les plus primées. Le second groupe est formé par les textes ou est indiquée la filiation de type latin et qui constitue la dernière phase de romanisation latine connue à Cárquere. Les formules rituelles y sont mieux employées. Nous retrouvons toujours ensemble HSE, STTL et FC sauf dans deux inscriptions (n. ${ }^{\circ} 37$ et 42 ). Ces dernières, commençant par HIC ou HS, sont ensuite coupées et le reste du texte n'est pas connu. Dans ce deuxième groupe, la formule DMS n'est jamais employée.

Les formules rituelles font donc partie du processus de latinisation de l'onomastique et des rites funéraires indigènes. La dernière étape de romanisation de cette onomastique témoigne d'un emploi mieux compris du système latin dans les épitaphes de Cárquere.

\section{Les structures familiales}

L'étude des structures familiales de la population de Cárquere s'avère assez courte $\mathrm{du}$ fait que les inscriptions nous fournissent très peu de renseignements sur ce sujet. Lorsque la filiation est indiquée, celle-ci se fait toujours par le père. Le rôle de la femme ne peut ainsi être déterminé dans la transmission du nom. En outre, les textes ne permettent jamais de reconstruire de stemma familial. Dans le cas cité plus haut, nous observons toujours la relation père-fils. Dans d'autres cas $(30 \%$ des textes), la personne ayant fait faire la stèle n'est jamais indiquée par son nom (sauf une fois), mais par sa parenté ou sa relation avec le défunt:

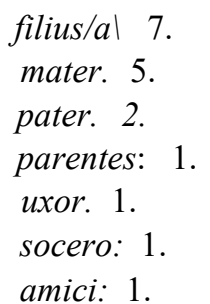

heres: 1

La seule exception est la stèle de Albonius, fils de Cumelius, faite par l'affranchi Viriatis (n. ${ }^{\circ} 32$ ). Les deux stèles de I. Fuscilla et $M$. Valerius Reburrus (n. ${ }^{\circ} 48$ et 49), retrouvées par V. Correia dans leur contexte funéraire, nous permettent de tenter de présenter le seul stemma suivant:

Conimbriga, 35 (1996) 69-106 
mater

Iulia Fuscilla
M. Valerius Reburrus

(époux)

\section{L'espérance de vie}

Sur les 63 stèles enregistrées, plus de la moitié (39) indiquent l'âge du défunt. Les quelques renseignements qui pourront être tirés à partir de l'âge à laquelle ces personnes sont décédées ne peuvent servir qu'à nous fournir une idée sur la moyenne d'âge de cette partie de la population de Cárquere. La découverte de nombreuses autres inscriptions dans ce village pourrait modifier légèrement ou totalement ces conclusions. Ces 39 textes nous permettent de présenter le tableau suivant:

moins de 20 ans 4

20-30 ans 8

30-40 ans $\quad 3$

40-50 ans $\quad 3$

$50-60$ ans 14

60-70 ans $\quad 4$

plus de 70 ans 3

Nous pouvons donc constater que la catégorie la plus importante est celle de 50-60 ans suivie de celle des 20-30 ans. Les personnes de plus de 50 ans (21) représentent $53,8 \%$, celles de 20 à 50 ans (14) constituent $35,9 \%$ et le moins de 20 ans ne sont que $12,3 \%$ (4).

L'âge minimum indiqué dans les épitaphes est de quinze ans. Jusqu'à présent, aucune stèle d'enfant n'a été retrouvée à Cárquere. L'âge le plus élevé est de 75 ans, si l'on ne tient pas compte de l'inscription $n .{ }^{\circ} 1$ posant des problèmes d'interprétation. Entre ces deux extrêmes, la moyenne d'âge de cette partie de la population connue par les inscriptions est de 44,5 ans. Toutefois, la découverte de nouvelles stèles peut faire augmenter ou diminuer cette moyenne.

\section{CONCLUSION}

Le village de Cárquere nous offre un ensemble épigraphique important avec la découverte d'au moins soixante-trois stèles funéraires. Aucun Conimbriga, 35 (1996) 69-106 
autre document épigraphique de type différent (autel, inscription honorifique, dédicace...) n'a, pour le moment, été retrouvé. Nous sommes donc en présence d'un contexte purement funéraire. Cet ensemble a malheureusement été dispersé au fil du temps et des découvertes et si une partie des monuments se retrouve dans les musées, l'autre partie reste non trouvée. Celles qui ont pu être conservées ont fait l'objet de quelques études mais c'est la première fois qu'un corpus est réalisé. Ce dernier, sûrement incomplet, a pu ainsi servir de base de donnée à une première étude sur l'art funéraire et la société de Cárquere.

La forme caractéristique des stèles et la répétition des mêmes décors nous incitent à localiser la présence d'un atelier de graveur, et peut-être de sculpteur, dans les environs proches du village. Le nombre important de stèles trouvées au même endroit ne laisse aucun doute sur la présence d'une nécropole des $\mathrm{II}^{\mathrm{e}}$ et $\mathrm{III}$ siècles ap. J.-C. en ce lieu ou deux sépultures ont seulement été trouvées. L'implantation d'une nécropole signifie l'existence proche d'un noyau d'habitation. Les quelques découvertes fortuites sur les pentes du mont de Cárquere laissent à penser à la présence d'un castro romanisé. L'hypothèse de la présence de ce castro est renforcée par une population indigène révélée par les épitaphes. Cette population aurait subi lentement les influences du dominant romain, au moins dans le cadre de l'épigraphie, liées au développement de la civilisation romaine dans la région et à l'habitude du latin.

L'existence d'un castro pré-romain, puis romanisé, ne peut être affirmé par la simple découverte de quelques vestiges archéologiques et par la losalisation d'une nécropole indigène. Seule une campagne sérieuse de prospections pourrait déterminer l'ampleur de la nécropole et localiser un noyau d'habitation de type castro sur les hauteurs. Ces prospections pourraient alors être suivies d'une campagne de fouilles qui constituerait la base d'une importante étude funéraire et épigraphique d'une localité indigène de l'extrême Nord de la Lusitanie dominant le fleuve Douro. Une étude de l'habitat et de la nécropole pourrait ainsi mettre en parallèle l'évolution de la romanisation du style de vie avec la romanisation des rites funéraires à Cárquere.

Conimbriga, 35 (1996) 69-106 


\section{ANNEXE I}

TABLEAU I:

Les différents décors des stèles.

\begin{tabular}{|c|c|c|c|c|c|c|c|c|c|}
\hline $\begin{array}{l}\text { Décor } \\
\text { Stèles }\end{array}$ & Fronton & Volutes & Rosace(s) & $\begin{array}{l}\text { Cercle(s) } \\
\text { gravé(s) }\end{array}$ & $\begin{array}{c}\text { Croissant } \\
\text { lunaire }\end{array}$ & Niche & Figure(s) & $\begin{array}{c}\text { Décor } \\
\text { latéraux }\end{array}$ & Anthropomorphe \\
\hline 1 & $\mathrm{X}$ & & $\mathrm{X}$ & & & & & $X$ & \\
\hline 3 & $X$ & & & $X$ & & & & $X$ & \\
\hline 4 & & & $X$ & & & & & $X$ & \\
\hline 6 & $X$ & $\mathrm{X}$ & & & $\mathrm{X}$ & & & & \\
\hline 7 & & & & & & & & & $X$ \\
\hline 8 & & & & & & & & & $\mathrm{X}$ \\
\hline 11 & $\mathrm{X}$ & & & $X$ & & & & & \\
\hline 15 & $X$ & & & & $X$ & & & $X$ & \\
\hline 17 & $X$ & $\mathrm{X}$ & & $X$ & & $\mathrm{X}$ & $X$ & & \\
\hline 18 & $X$ & & & $X$ & & & & & $\mathrm{X}$ \\
\hline 19 & $\mathrm{X}$ & & & & & $X$ & $X$ & & \\
\hline 20 & $?$ & $\mathrm{X}$ & $\mathrm{X}$ & & & & & & \\
\hline 21 & $X$ & $\mathrm{X}$ & & & & $\mathrm{X}$ & $\mathrm{X}$ & $X$ & \\
\hline 24 & & & $\mathrm{X}$ & & & & & $X$ & \\
\hline 26 & & & & & & & & $X$ & \\
\hline 27 & $\mathrm{X}$ & $X$ & & $\mathrm{X}$ & & & & $X$ & \\
\hline 32 & $X$ & & & & & & & $X$ & \\
\hline 33 & $?$ & $\mathrm{X}$ & & & & $\mathrm{X}$ & $\mathrm{X}$ & $\mathrm{X}$ & \\
\hline 35 & & $X$ & & $\mathrm{X}$ & & & & $X$ & \\
\hline 36 & 7 & & & $X$ & & & & $\mathrm{X}$ & \\
\hline 37 & 7 & & & $\mathrm{X}$ & & & & $\mathrm{X}$ & \\
\hline 38 & & & $\mathrm{X}$ & & & & & $\mathrm{X}$ & \\
\hline 39 & $\mathrm{X}$ & $\mathrm{X}$ & $X$ & & & & & $\mathrm{X}$ & \\
\hline 40 & 7 & & & & $\mathrm{X}$ & & & & \\
\hline 42 & $X$ & & & & & & & & \\
\hline 43 & & & & & & & & $X$ & \\
\hline 45 & $X$ & $X$ & & & $X$ & & & $X$ & \\
\hline 46 & $X$ & & & $\mathrm{X}$ & & & & $\mathrm{X}$ & \\
\hline 47 & & & & & & & & $X$ & \\
\hline 48 & & & $\mathrm{X}$ & & 7 & & & & \\
\hline 50 & $\mathrm{X}$ & & $\mathrm{X}$ & & & & & $\mathrm{X}$ & \\
\hline 52 & & & & & & $\mathrm{X}$ & $X$ & & \\
\hline 54 & & & & & & & & & $X$ \\
\hline 58 & $\mathrm{X}$ & & & & & $\mathrm{X}$ & $?$ & & \\
\hline 59 & $\mathrm{X}$ & & & & & & $X$ & & \\
\hline 63 & & & & & & & & $X$ & \\
\hline Total & $\begin{array}{c}18 \\
(23 ?)\end{array}$ & 9 & 8 & 9 & $4(5 ?)$ & 6 & $6(7 ?)$ & 22 & 3 \\
\hline
\end{tabular}

Conimbriga, 35 (1996) 69-106 
TABLEAU II:

Les symboles astraux sur les stèles de Cárquere.

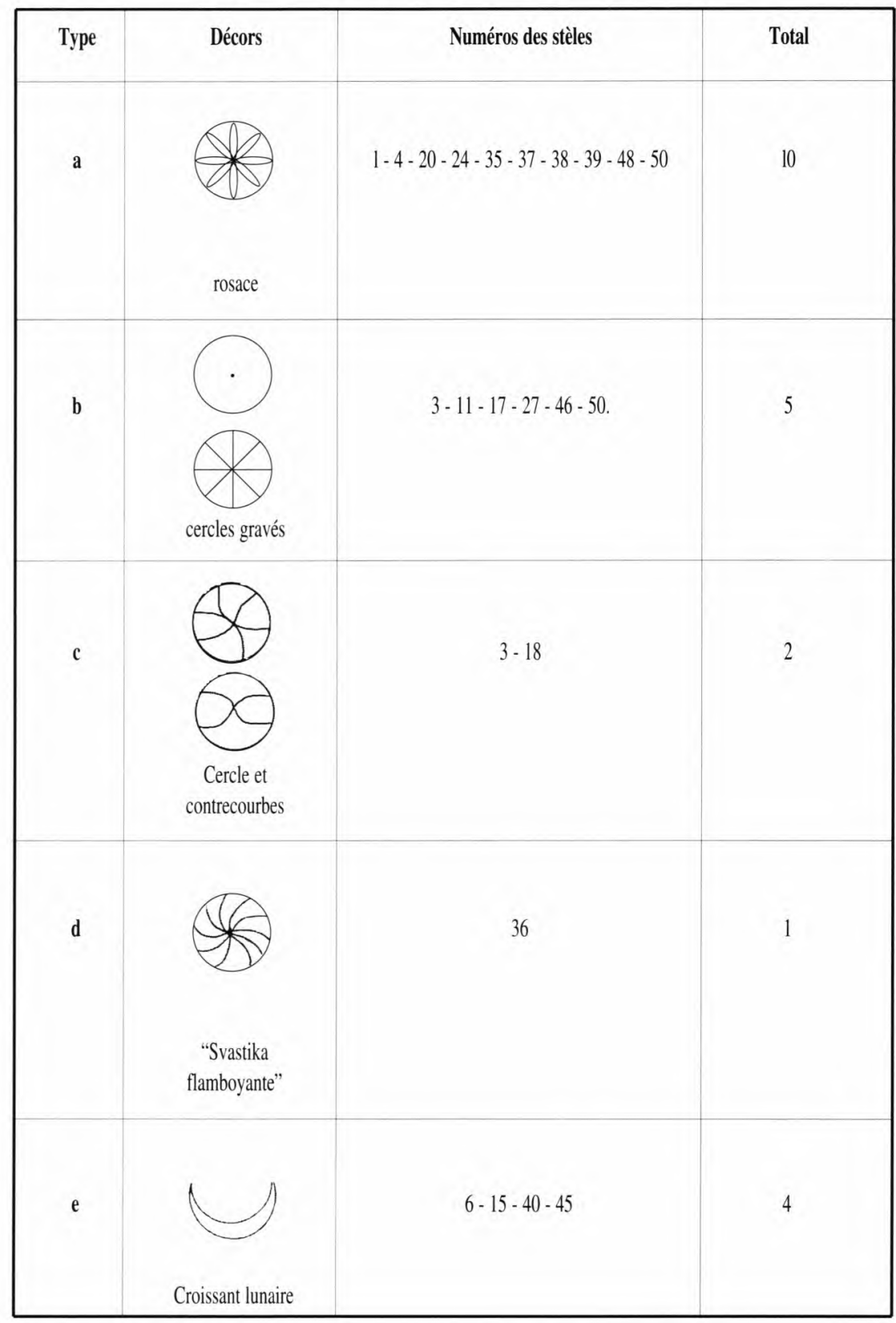

Conimbriga, 35 (1996) 69-106 


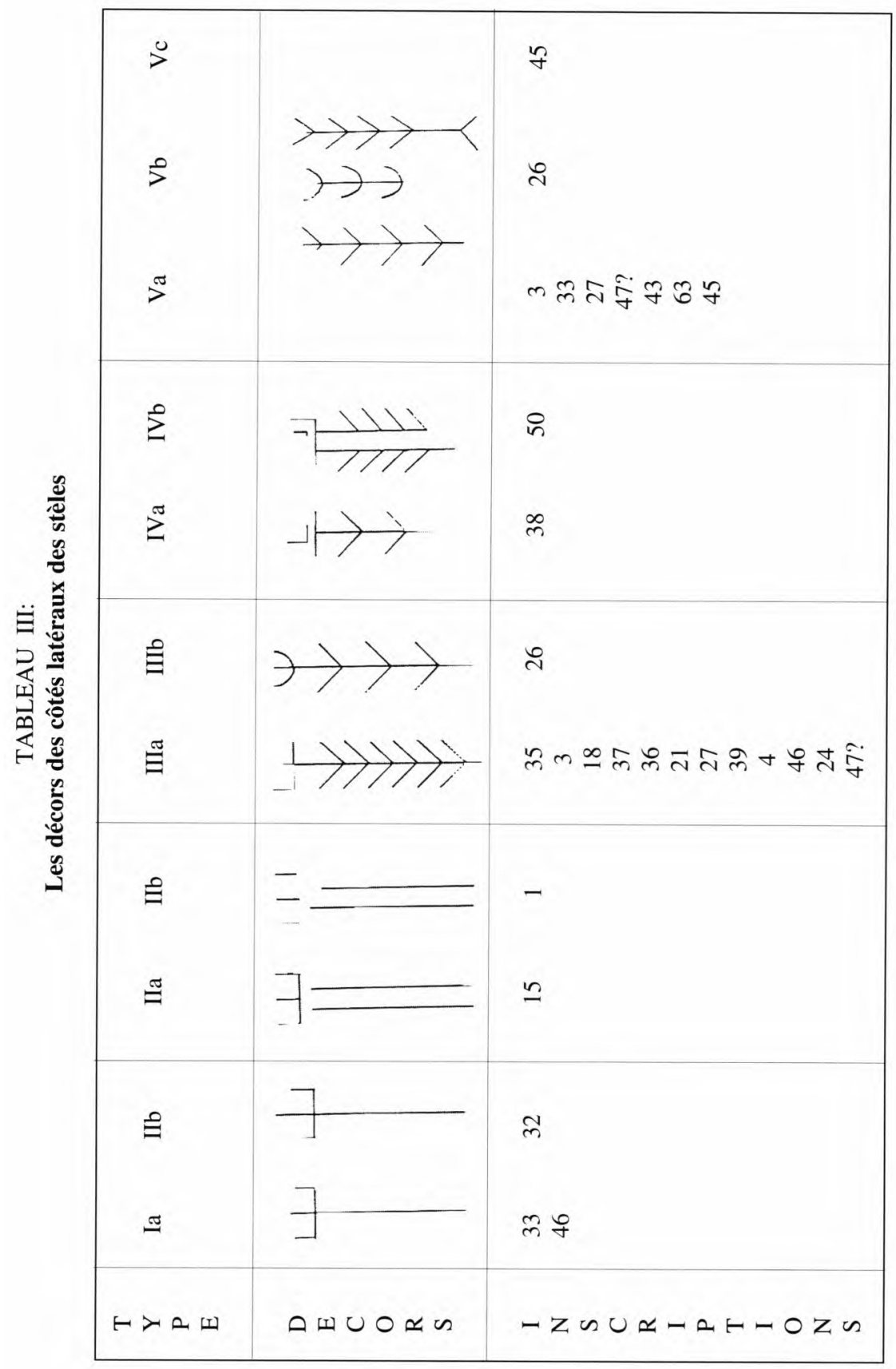

Conimbriga, 35 (1996) 69-106 


\section{ANNEXE II}

\section{LES STELES}

1. CÁRQUERE. Fronton de stèle

Bibl: Vaz, 1982, n. ${ }^{\circ}$ 10, photo 10, p. 509.

2. CÁRQUERE. Epitaphe de Af...? Amoena '?

$\mathrm{D}$ (iis) $\quad \mathrm{M}$ (anibus)/S(acrum) $\mathrm{AFA}($ ?) $\quad \mathrm{AM}$ (moenae ?)*A(nnorum)/LXXV

Consacré aux dieux Manes et à Afa(...) Amoena?, âgée de soixante-quinze ans. Bibl.: Vasconcellos, 1888, pp. 114, 1900, p. 207; C. 1. L. II, 5575

3. CÁRQUERE. Epitaphe de Afinatus

AFINATV(s)/F(ilio) $・ \mathrm{~A}($ nnorum $) \cdot \mathrm{XX} \cdot \mathrm{S}(\mathrm{it}) \cdot \mathrm{T}(\mathrm{ibi}) \cdot \mathrm{T}($ erra $) / \mathrm{L}($ evis $) \cdot \mathrm{M}($ ater $)$ - $\mathrm{F}$ (aciendum) C(uravit)

Cit gît Afinatus, âgée de vingt ans. Que la terre te soit légère. La mère a pris soins d'élever ce monument à son fils

Bibi: Dias, 1986, pp. 190-193, fig. 2.

4. CÁRQUERE. Epitaphe de Albonius

ALBONI/VS CVM/ELI(i) - F(ilius) - AN(norum)/LX • H(ic) - S(itus) • EST/

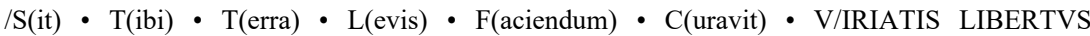

Cit gît Albonius, fils de Cumelius, âgé de soixante ans, que la terre te soit légère, l'affranchi Viriatis (ou de Viriatis) a pris soins d'élever ce monument.

Bibl: Jalhay, 1951, pp. 77-79.

5. CÁRQUERE. Epitaphe de Albura

[- - -]/ALB/VRA/[- - ]

Bibl: Mattos, 1948, p. 65, n. ${ }^{\circ} 81$; Dias, 1987, p. 196, note 8.

6. CÁRQUERE. Epitaphe de Amena

$\mathrm{D}$ (iis) $\quad \mathrm{M}$ (anibus) $\quad \mathrm{S}$ (acrum)/AM(o)E(nae) $\quad \mathrm{RVF}$ (inae?) $\quad \mathrm{CE}$ (liae?) vel $\mathrm{CE}$ (lti?) (filiae)/A(nnorum) XXXV

Consacré aux dieux Manes de Amoena Rufina?, fille de Cellea ou de Celtus, âgée de trente-cinq ans.

Bibl.: inédite

7. CÁRQUERE. Epitaphe de Amoena

$\mathrm{D}$ (iis) $\cdot \mathrm{M}$ (anibus) $\cdot \mathrm{S}($ acrum $) / \mathrm{F}(?) \cdot \mathrm{AM}(\mathrm{o}) \mathrm{E} / \mathrm{NA}(\mathrm{e}) \cdot($ annorum $) \mathrm{LXI}$

Consacré aux dieux Manes. Cit gît $\mathrm{F}(. .$.$) Amoena, âgée de soixante et un ans.$ Bibl: Sarmento, 1887, p.186; Cardozo, 1985, n. ${ }^{\circ}$ 51, p. 93.

8. CÁRQUERE. Epitaphe de Amoena?

$\mathrm{D}$ (iis) $\quad \mathrm{M}$ (anibus) $\quad \mathrm{S}$ (acrum) $\quad \mathrm{F}$ (iliae?) $\quad$ vel $\quad \mathrm{F}($...)/AM(oenae?) $\quad \mathrm{A}$ (nnorum)/

/[?]XXCII[?]

Conimbriga, 35 (1996) 69-106 
Consacré aux dieux Manes et à ma fille Amoena, âgée de 83 ans?

$\mathrm{Ou}$

Consacré aux dieux Manes et à Amoena, âgée de 83 ans?

B ibi: Vasconcellos, 1888, pp. 114; 1900, p. 211; 1913, p. 456; C. 1.L. II 5574.

9. CÁRQUERE. Epitaphe de Amoena?

DIBVS/M(anibus) S(acrum) F(iliae?) vel F(...) A/M(oenae?)

Consacré aux dieux Manes et à ma fille Amoena?

$\mathrm{Ou}$

Consacré aux dieux Manes et à $F(. .$.$) Amoena?$

B ibi: Jalhay, 1951, pp. 74-75.

10. CÁRQUERE. Epitaphe de Amoena

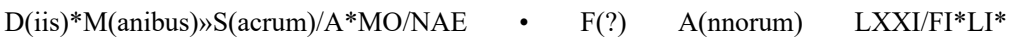
$<0>$ \{us $\}$ F(ecit)

Inscription enregistrée au Musée National d'Archéologie de Lisbonne et dont la stèle reste non trouvée.

11. CÁRQUERE. Epitaphe de Aurelius

D(iis) $\quad \mathrm{M}$ (anibus) $\quad \mathrm{S}$ (acrum) $\quad \mathrm{D}$ (edicatum)/AVR(elius) vel $\quad$ AVR(elii) vel AVR(elio) RVF(us) vel RVF(i) vel RVF(o)

Consacré et dédié aux dieux Manes, (cit gît) Aurelius Rufus ou fils de Rufus.

Consacré et dédié aux dieux Manes et à Aurelius Rufus ou fils de Rufus.

Consacré et dédié aux dieux Manes de Aurelius Rufus ou fils de Rufus.

Bibi: Vasconcellos, 1900, pp. 210-211.

12. CÁRQUERE. Epitaphe de Avitus Gracilis

AVITVS/GRACILI/S • H(ic) • S(itus) vel AVITVS/GRACILI(s)/S(ervus) H(ic) S(itus)

Cit gît Avitus Gracilis.

$\mathrm{Ou}$

Cit gît Avitus, esclave de Gracilis.

Bibi: Sarmento, 1887, p. 186; Cardozo, 1985, n. ${ }^{\circ}$ 58, p. 100.

13. CÁRQUERE. Epitaphe de Bas... $\mathrm{L}(\mathrm{evis})[---]$

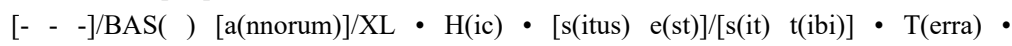

Cit gît ( ) Bas.., âgé/e de quarante ans, que la terre te soit légère

$B$ ibi: inédite

14. CÁRQUERE. Epitaphe de Cainus?

CAINO/[C'?] ATV(ri vel ronis) F(ilio)

(Cit gît) Cainus?, fils de Caturo ou de Caturonis.

B ibi.: inédite

Conimbriga, 35 (1996) 69-106 
15. MEIJINHOS. Epitaphe de Cellea

CELLEA CEL/TG) • F(ilia) • ANN(orum)/XVIII/PARENTES/F(aciendum)

$\mathrm{C}($ uraverunt $) \cdot \mathrm{H}($ ic $) \cdot \mathrm{S}($ itus $) \cdot \mathrm{E}(\mathrm{st}) \cdot \mathrm{SGt}) / \mathrm{T}($ ibi $) \cdot \mathrm{T}($ erra $) \cdot \mathrm{L}($ evis $)$

Cit gît Cellea, fille de Celtus, âgée de 18 ans, ses parents ont pris soins d'élever ce monument, que la terre te soit légère

Bibl: Vaz, 1983, pp 579-580

16. CÁRQUERE. Epitaphe de Cellea?

$\mathrm{P}($ ?)/D(iis) $\mathrm{M}$ (anibus)/S(acrum) CEL/I(eae)

Consacré aux dieux Manes de/et à Cellea.

Bibl.: Jalhay, 1951, pp 80-81

17. CÁRQUERE. Epitaphe de Cellea?

D(iis) $\quad \mathrm{M}$ (anibus) $\quad \mathrm{S}$ (acrum)/CEL(leae?) $\quad \mathrm{AN}(\mathrm{n}) 0($ rum $) / \mathrm{LXV}$

Consacré aux dieux Manes et a Cellea?, âgée de soixante-quinze ans

Bibl.: inédite

18. CÁRQUERE. Epitaphe de Iboena

IBOENA/ALLVQVI0) FGlia)/AN(norum) XXXV ET/AV*IT*V*S F/

/RONTONIS/AN(norum) XLII

Cit gisent Iboena, fille de Alluquius, âgée de 35 ans et Avitus, fils de Fronto, âgé de 42 ans

Bibl: Dias, 1986, pp 192-194, fig 3

19. CÁRQUERE. Epitaphe de Iulia Fuscilla

$\mathrm{D}$ (iis)*M(anibus)»S(acrum)/IVLIAE FVS/CILLAE AN(norum) XXX/

/MATER $» \mathrm{~F}$ (aciendum) $\mathrm{C}$ (uravit)

Consacré aux dieux Manes et à Iulia Fuscilla, âgée de trente ans, sa mère a pris soins d'élever ce monument.

Bibl.: Mattos, 1948, p. 68, n. ${ }^{\circ} 84$; Correia, 1972, pp. 261-263.

20. CÁRQUERE. Epitaphe de Iulia Sunua

$\mathrm{D}$ (iis) - $\mathrm{M}$ (anibus) - $\mathrm{S}($ acrum)/I(ulia) - SVNVA/LIBERTA/ANNORVM/LV •

FILIA/[P(onendum)] vel [F(aciendum)] C(uravit)

Consacré aux dieux Manes, (Cit gît) Iulia Sunua, affranchie, âgée de 55 ans, sa fille à fait poser ou faire ce monument.

Bibl.: Vasconcellos, 1913, p. 414.

21. CÁRQUERE. Epitaphe de Iulia Tongeta

$\mathrm{D}$ (iis) $-\mathrm{M}$ (anibus) $・ \mathrm{~S}$ (acrum)/IVLIA TONGETA/AN(norum) XXXXTM(ater)

- F(aciendum) C(uravit)

Consacré aux dieux Manes. (Cit gît) Iulia Tongeta, âgée de 40 ans, sa mère a pris soins d'élever ce monument.

Bibl.: Vasconcellos, 1929, pp. 209-227.

Conimbriga, 35 (1996) 69-106 
22. CÁRQUERE. Epitaphe de Iunius

IVNIVS/PINTAM/I $\cdot \mathrm{F}$ (ilius) $\cdot \mathrm{AN}($ norum) $\cdot \mathrm{L} / \mathrm{H}($ ic) $\cdot \mathrm{S}($ itus $) \cdot \mathrm{E}(\mathrm{st}) \cdot \mathrm{S}(\mathrm{it}) /$ $/ \mathrm{T}($ ibi $) \cdot \mathrm{T}($ erra $) \cdot \mathrm{L}($ evis $) \cdot \mathrm{F}$ (ilius) $\bullet \mathrm{F}$ (aciendum) $\bullet \mathrm{C}$ (uravit)

Cit gît Iunius, fils de Pintamus, âgé de cinquante ans, que la terre te soit légère, son fils a pris soins d'élever ce monument.

Bibi: Dias, 1989, p. 381, n. ${ }^{\circ}$ 63; Vaz, 1986, p.285-293 et 304.

23. CÁRQUERE. Epitaphe de Maelia Rufina

MAELI $\{a e\} / R V F I N\{a\} E / A N($ norum) $\quad$ XXX/M(ater) F(aciendum) $\quad$ C(uravit)

A Maelia Rufina, âgée de trente ans, sa mère a pris soins d'élever ce monument.

Bibl: Jalhay, 1951, pp. 83-84.

24. CÁRQUERE. Epitaphe de Mailgenus?

[M]AILG/[e]NVS/[C]VME/[li(i) f(ilius)]

(Cit gît) Mailgenus, fils de Cumelius.

Bibi: Dias, 1989, p. 382, n. ${ }^{\circ} 64$.

25. CÁRQUERE. Epitaphe de M. Valerius Reburrus

$\mathrm{M}(\operatorname{arco}) \cdot \mathrm{VAL}($ erio) RE/BVR $\cdot \mathrm{RO} \cdot \mathrm{AN}($ norum)/L $\cdot \mathrm{V} \cdot \mathrm{IVLIA} / \mathrm{FVSCILL} / \mathrm{A}$

- SOCERO/F(aciendum) $・ \mathrm{C}($ uravit) $\cdot \mathrm{H}(\mathrm{ic}) \cdot \mathrm{S}($ itus $) \cdot \mathrm{S}(\mathrm{it}) \cdot \mathrm{T}(\mathrm{ibi}) \cdot \mathrm{T}($ erra $) \cdot \mathrm{L}($ evis $)$

A son beau-père, Marcus Valerius Reburrus, âgé de cinquante-cinq ans, Iulia Fuscilla à pris soins d'élever ce monument. Il gît ici. Que la terre te soit légère.

Bibl: Mattos, 1948, p. 68, n. ${ }^{\circ} 84$; Correia, 1972, pp. 261-263.

26. CÁRQUERE. Epitaphe de Melia Toceta

MELIA7TOCETA/A(nnorum) $・ \mathrm{XXV} \cdot \mathrm{F}($ ecit?)/M(ater?) $・ \mathrm{C}$ (onsecravit) $・ \mathrm{~T}$ (itulus)

(Cit git?) Melia Toceta, âgée de vingt-cinq ans, sa mère a pris soins d'élever et de consacrer cette inscription?

Bibl: Vasconcellos, 1888, pp. 114; 1900, pp. 211-212; C. 1. L. II, 5576.

27. CÁRQUERE. Epitaphe de Occarius

$\mathrm{D}($ iis) $) \mathrm{M}($ anibus)*S(acrum)/OCCARII/AN(norum)«+ + +/FAC(iendum) CVR(avit)

Consacré aux dieux Manes de Occarius, âgé de vingt ans. Il a fait faire ce monument.

Bibl: Vasconcellos, 1929, pp. 220.

28. CÁRQUERE. Epitaphe de ?

OVINA/[ann]OR(um) II S(it) T(erra) L(evis)

(Cit gît) Ovina, âgée de deux ans, que la terre (lui) soit légère.

Bibl: Jalhay, 1951, p. 84.

29. CÁRQUERE. Epitaphe de Pentilius

PENTILI(i)/CAMALI F(ilii)/ANNORV/M LXX

Conimbriga, 35 (1996) 69-106 
A Pentilius, fils de Carnalius, âgé de 70 ans.

BibL: Dias, 1986, pp. 194-195, 1989, p. 381.

30. CÁRQUERE. Epitaphe de Pentilius

D(iis) $\quad \mathrm{M}$ (anibus) $\quad \mathrm{S}$ (acrum)/PEN(tilio)/AN(norum)/LXXXI

Consacré aux dieux Manes et à Pentilius, âgé de 81 ans.

BibL: Dias, 1986, pp. 199-200, fig. 8.

31. CÁRQUERE. Epitaphe de Pissirea

[P]ISSI/REA/TANCI/NI F(ilia)

A Pissirea, fille de Tancinus

BibL: Dias, 1986, pp. 198-199, fig 7.

32. CÁRQUERE. Epitaphe de Pissirus

PISSIRVS/MEBDI $・ F($ ilius)/AN(norum) $\bullet \mathrm{XV} \bullet \mathrm{H}($ ic) $/ \mathrm{S}($ itus $) \bullet \mathrm{E}(\mathrm{st}) \cdot \mathrm{S}(\mathrm{it})$

$\cdot \mathrm{T}(\mathrm{ibi}) \cdot \mathrm{T}($ erra $) \cdot \mathrm{L}($ evis $) / \mathrm{P}($ ater $) \cdot \mathrm{F}($ aciendum $) \cdot \mathrm{C}$ (uravit)

Cit gît Pissirus, fils de Mebdus, âgé de quinze ans, que la terre te soit légère, le père à pris soins d'élever ce monument.

BibL: Sarmento, 1887, p. 186-187; Cardozo, 1985, n. ${ }^{\circ} 49$, p. 91.

33. CÁRQUERE. Epitaphe de Ponius?

$\mathrm{D}$ (iis) - $\mathrm{M}$ (anibus) - $\mathrm{S}($ acrum)/PON(io/a) - IVNI(i) (filio vel filia)/AN(norum) $\bullet$ LUI

Consacré aux dieux Manes et à Ponius/Ponia, fils/fille de lunus, âgé(e) de 53 ans. BibL: Vasconcellos, 1913, pp. 454-455.

34. CÁRQUERE. Epitaphe de Promut

PROMVT< us $>$ ?/AN(norum) $\bullet \mathrm{C} \bullet \mathrm{H}($ ic $) \cdot \mathrm{S}($ itus $) /[---]$

Cit gît Promulus?, âgé de cent ans.

BibL: Vaz, 1982, n. ${ }^{\circ}$ 7, p. 509; C.7.L., II, 5253.

35. CÁRQUERE. Epitaphe de Rufinus

RVFINVS/CALAET(i) (filius)• AN(norum)/LX (mensium) $\ll V \quad$ (dierum) $\ll \mathrm{X}^{*}$ $\mathrm{A}($ mici $) \cdot \mathrm{F}($ aciendum $) / \mathrm{C}($ uraverunt $) \cdot \mathrm{D}($ iis $) \cdot \mathrm{M}($ anibus $) \cdot \mathrm{S}($ acrum $) / \mathrm{S}($ it $) \cdot \mathrm{T}($ ibi $) \cdot \mathrm{T}($ erra $)$

$\cdot \mathrm{L}($ evis $) \cdot \mathrm{H}($ ic) $)$ C (cubat) vel $\mathrm{H}($ ic) $)$ C(rematus est)

Ici est incinéré Rufinus, fils de Calaetus, âgé de soixante ans, cinq mois, dix jours, ses amis ont pris soins d'élever ce monument, consacré aux dieux Manes. Que la terre te soit légère.

BibL: Dias, 1986, pp 189-190, fig. 1.

36. CÁRQUERE. Epitaphe de Rufinus

RVFINV[s]/RVFI $• \mathrm{~F}$ (ilius) $\bullet \mathrm{A}[\mathrm{n}$ (norum)]/[- - ]

(Cit gît) Rufinus, fils de Rufus, âgé de ...

BibL: Dias, 1986, pp. 197-198, fig. 6.

Conimbriga, 35 (1996) 69-106 
37. CÁRQUERE. Epitaphe de Rufus RVFV(s)/RVFI F(ilius)/AN(norum)?/HIC [s(itus)] [e(st)]

Cit gît Rufus, fils de Rufus.

B ibi : Dias, 1986, pp. 195-197, fig. 5.

38. CÁRQUERE. Epitaphe de Rufus

S(acrum) M(anibus) D(iis)/RVFVS

Consacré aux dieux Mânes, (Cit gît) Rufus.

B ibi: Vasconcellos, 1900, p. 209-210.

39. CÁRQUERE. Epitaphe de Saturninus

D(iis) M(anibus) S(acrum)/SATVR/NINVS/ CLEME/[ns] vel CLEMEfntis]

Consacré aux dieux Manes, (Cit gît) Saturninus Clemens.

$\mathrm{Ou}$

Consacré aux dieux Manes, (Cit gît) Saturninus fils de Clemens.

Bibl: Vasconcellos, 1900, p. 209.

40. CÁRQUERE. Epitaphe de Severus

[Se]VERVS/[0]CVLATI - F(ilius)/ANNORVM $-\mathrm{XL} \cdot \mathrm{H}(\mathrm{ic}) \cdot \mathrm{S}$ (itus) $\cdot \mathrm{E}(\mathrm{st})$

$[\mathrm{s}(\mathrm{it})] / \mathrm{T}(\mathrm{ibi}) \cdot \mathrm{T}($ erra $) \cdot \mathrm{L}($ evis $) \cdot \mathrm{F}$ (ilius) $\bullet \mathrm{F}$ (aciendum) $\bullet \mathrm{C}$ (uravit)

Cit gît Severus, fils de Oculatus, âgé de soixante ans, que la terre te soit légère, son fils a pris soins d'élever ce monument.

Bibl: Jalhay, 1951, p. 76-77.

41. CÁRQUERE. Epitaphe de Sunua

SVNVA/CASABI F(ilia)/AN(norum) $\bullet$ LX

(Cit gît) Sunua, fille de Casabus, âgée de soixante ans.

Bibl.: Vasconcellos, 1888, pp. 115; 1900, p. 210; CIL II, 5577.

42. CÁRQUERE. Epitaphe de Tolia Peda

TOLIA/PEDA/CARI $\bullet$ F(ilia)/AN(norum) $\bullet$ L $\bullet \mathrm{H}$ (ic) $\bullet$ S(ita)

Cit gît Tolia Peda, fille de Carus, âgée de cinquante ans.

Bibl: Jalhay, 1951, pp. 75-76.

43. CÁRQUERE. Epitaphe de Tongeta

[D(iis) $] \quad \mathrm{M}$ (anibus) $\quad \mathrm{S}$ (acrum)/TONGETAE/RVFI (filiae) $\quad$ AN(norum) LXX vel IXX

Consacré aux dieus Manes et à Tongeta, fille de Rufus, âgée de soixante dix ou dix-neuf ans.

Bibl.: Dias, 1989 , p. 382, n. $^{\circ} 65$.

44. CÁRQUERE. Epitaphe de Ulpius Sabinus

VLPIVS SA/BINVS AN(norum)/[-]I $\cdot \mathrm{H}(\mathrm{ic}) \cdot \mathrm{S}(\mathrm{itus}) \cdot \mathrm{E}(\mathrm{st}) \cdot \mathrm{S}(\mathrm{it}) \cdot \mathrm{T}(\mathrm{ibi}) /$

$/[\mathrm{t}(\mathrm{erra}) 1(\mathrm{evis})]$

Conimbriga, 35 (1996) 69-106 
Cit gît Vlpius Sabinus, âgé de ? ans, que la terre te soit légère.

Bibl.: Vasconcellos, 1900, pp. 207-208.

45. CÁRQUERE. Epitaphe de Valeria Amoena

$\mathrm{D}$ (iis) $\bullet \mathrm{M}$ (anibus) $\bullet \mathrm{S}($ acrum $) / \mathrm{VAL}($ eriae $) \cdot \mathrm{AMOE} / \mathrm{NAE}$ A(nnorum) LXX/FI

- $\mathrm{LI} \bullet<0>\{$ us $\} \cdot \mathrm{F}($ ecit $)$

Consacré aux dieux Manes et à Valeria Amoena, âgée de soixante-dix ans, son fils a fait (élever ce monument).

Bibl.: inédite.

46. CÁRQUERE. Epitaphe de Valerius Reburrus (1).

$\mathrm{D}$ (iis) - $\mathrm{M}$ (anibus) - $\mathrm{S}$ (acrum)/VAL(erius) RE/BVRRVS/ANNO(rum) •

$\mathrm{L} / \mathrm{F}$ (ilius) $\bullet \mathrm{F}$ (aciendum) [c(uravit)]

Consacré aux dieux Manes, (Cit gît) Valerius Reburrus, âgé de cinquante ans, son fils a pris soins d'élever ce monument.

Bibl.: Jalhay, 1951, pp. $81-82$.

47. CÁRQUERE. Epitaphe de Valerius Reburrus (2).

[V]AL(erio) RE/[bu]RRO/[- - - ]

A Valerius Reburrus.

Bibl.: Reigota, 1989, n. ${ }^{\circ} 14$; Dias, 1992, p. 471, n. ${ }^{\circ} 172$.

48. CÁRQUERE. Epitaphe de Viriatis

[V]IRIATIS/[S]EVERI F(ilius)/A • N(norum) • XXX ?

Viriatis, fils de Severus, âgé de trente ans ?.

Bibl.: Jalhay, 1951, p. 79-80.

49. CÁRQUERE. Epitaphe de ?

[- - - ]/XXV[- - ]/RAIITIVF/RN[ -]VT TONGETO/ARANTO/[- ]

Inscription enregistrée au Musée National d'Archéologie de Lisbonne et dont la stèle reste non trouvée.

50. CÁRQUERE. Epitaphe de C...

$\mathrm{D}($ iis $) \cdot \mathrm{M}($ anibus $) \cdot \mathrm{S}($ acrum $) / \mathrm{C}($ aii/o?) $\bullet \mathrm{C}($ aii?) $\bullet \mathrm{F}($ ilio? $) \cdot \mathrm{R}($ ufi/o)/AN(norum)

$\cdot \mathrm{LV} / \mathrm{F}($ ilius $) \cdot \mathrm{F}($ aciendum $) \cdot \mathrm{C}($ uravit $) / \mathrm{S}($ it $) \cdot \mathrm{T}($ ibi $) \cdot \mathrm{T}($ erra $) \cdot \mathrm{L}($ evis $) / \mathrm{C} \cdot \mathrm{C} \cdot \mathrm{M}$

Consacré aux dieux Manes de/et à Caius? Rufus?, fils de Caius?, âgé de 55 ans.

Son fils a pris soins d'élever ce monument.

Bibl.: Vasconcellos, 1929, pp. 215-216.

51. CÁRQUERE. Epitaphe de ?

RVT?A[- - ]/RVIIV[- - - ]/IIAN[- - - ]/F • F

Inscription enregistrée au Musée National d'Archéologie de Lisbonne et dont la stèle reste non trouvée.

Conimbriga, 35 (1996) 69-106 
52. CÁRQUERE. Epitaphe de ?

$\mathrm{D}$ (iis) $\mathrm{M}$ (anibus) $/[-] \mathrm{ON}[-] \quad$ (et) $[-] / \mathrm{AN}($ norum) $\quad \cdot \mathrm{XX}$ (et) $\mathrm{IV}[-] / \mathrm{ASSV}(?)$

Au dieux Manes de (on...), âgé/e de vingt ans et de (...), âgé/e de (...)ans ?

B ibi: Vasconcelos, 1888, pp. 115; 1900, p. 210; C. /. L. II, 5579.

53. CÁRQUERE. Epitaphe de ...tai...nus

[ - - ]TAI[- - - ]/[- - -]NVS[- - -]/[- - -]LACO[brigens]/[- - -]AN(norum) XV[- - -]

(Cit gît) (...)tai(...)nus, habitant de Lacobriga, âgé de XV... ans.

Bibl: Reigota, 1989, n. ${ }^{\circ}$ 148; Dias, 1992, p. 472, n. ${ }^{\circ} 173$.

54. CÁRQUERE. Epitaphe d'un fils au père

$\mathrm{D}$ (iis) $\quad \mathrm{M}$ (anibus) $\quad \mathrm{S}$ (acrum)/F(ilio) $\quad \mathrm{P}($ ater) $\quad \mathrm{AN}$ (norum) $/ \mathrm{XXXIII}$

Consacré aux dieux Manes, à son fils, âgé de trente trois ans, le père (a pris soins d'élever ce monument).

Bibl: Jalhay, 1951, p. 74

55. CÁRQUERE. Epitaphe de ?

[------]XI H(ic)/[s(itus) e(st)] S(it) T(ibi) T(erra) L(evis) A.../[------------------ ] VX(or)

$\mathrm{F}$ (aciendum) $\mathrm{C}$ (uravit)

Cit gît..., âgé de ...XI ans, que la terre te soit légère, A..., sa femme, a pris soins d'élever ce monument.

Bibl: Dias, 1989, pp. 373-384.

56. CÁRQUERE. Epitaphe de ?

$[------]$ AN(norum) $\bullet X X /[-----------] ~[H(i c)] ~ S(i t u s) \cdot E(s t) \bullet S(i t) \bullet T(i b i) \cdot T($ erra $)$

- L(evis)

Cit gît..., âgé de vingt ans, que la terre te soit légère.

Bibl: Jalhay, 1951, pp. 84-85.

57. CÁRQUERE. Epitaphe de F...

$\mathrm{D}$ (iis) $\mathrm{M}$ (anibus) $\mathrm{S}$ (acrum) $\quad \mathrm{F}(\ldots) / \mathrm{FV}(\ldots) \quad \mathrm{A}$ (nnorum) $\quad \mathrm{XXX}$

Consacré aux dieux Manes de $\mathrm{F}(\ldots) \mathrm{Fu}(. .$.$) , âgé de trente ans.$

Bibl: inédite

58. CÁRQUERE. Epitaphe de ?

$\mathrm{DI}($ is) $\mathrm{MA}$ (nibus)/SAC(rum) $• \mathrm{~F}$ (?) A/[moenae] vel F(ilio) A(nnorum)/[-------------------]

Consacré aux dieux Manes de $\mathrm{F}(. .$.$) Amoena?$

ou

Consacré aux dieux Manes et à (mon) fils, âgé de ... ans?

Bibl: inédite

59. CÁRQUERE. Epitaphe de ?

$\mathrm{D}$ (iis) $\mathrm{M}$ (anibus) $\mathrm{S}$ (acrum) $\quad$ (vinae?)/R(ufi?) $\mathrm{F}$ (filiae) $\mathrm{AN}$ (norum) $\mathrm{L}$

Consacré aux dieux Manes de Ovina, fille de Rufus (ou dérivé)?, âgé de cinquante ans.

Bibl: inédite 
60. CÁRQUERE. Epitaphe de ?

[-----]TI $•$ F $•$ F?[-------]/[----- ] AN L

Inscription enregistrée au Musée National d'Archéologie de Lisbonne et dont la stèle reste non trouvée.

61. CÁRQUERE. Epitaphe de [- - - ]s

[--- ]/[-]S A(nnorum)/LX (diebus?)V/A

Cit gît...., âgé de soixante ans et cinq mois, A...?

Bibl: inédite

62. CÁRQUERE. Epitaphe de ?

$[-$ - $] / \mathrm{S}($ itus $) \cdot \mathrm{E}(\mathrm{st}) \cdot \mathrm{S}(\mathrm{it})[\mathrm{t}(\mathrm{ibi}) \mathrm{t}($ erra $)] / \mathrm{L}($ evis $) \cdot \mathrm{H}($ eres $) \cdot \mathrm{F}$ (aciendum) $[\mathrm{c}($ uravit)]

Cit gît..., que la terre te soit légère, l'héritier a pris soins d'élever ce monument.

Bibi: Dias, 1986, p. 202, fig. 9.

63. CÁRQUERE. Epitaphe de ?

$[-----] / A N($ norum $) \cdot \mathrm{IXX} /[--------]$

Cit gît..., âgé de dix-neuf ans.

Bibi: Dias, 1989, p. 383, n. $^{\circ} 70$.

\section{BIBLIOGRAPHIE}

Albertos 1976: Albertos (M. L.). - "La antroponimia prerromana de la Peninsula Ibérica", Actas del I Coloquio sobre Linguas y Culturas prerromanas de la Peninsula Iberica, Salamanca 27-31 Maio 1974, Salamanca, 1976.

Borges de Figueiredo 1888: Borges DE Figueiredo. - "Inscrições de Lamego e de Quiniela de Penude", Revista Archeologica e Histórica, II, 1888, p. 172.

Camoy 1906: CaRnoy (A.). - Le latin d'Espagne d'après les inscriptions, Bruxelles, 1906.

Cardozo 1972: CARdozo (M.). - Catálogo do Museu da Sociedade Martins Sarmento. Secção de epigrafia latina e de escultura antiga, $3 .^{\text {a }}$ ed., 1985, p. 91, n. ${ }^{\circ} 49 ;$ p. 93, n. ${ }^{\circ} 51 ;$ p. 100, n. $^{\circ} 58$.

Cordeiro Laranjo 80/81 : CoRdeiro LARANJo (F. J.). - "A igreja de Santa Maria de Almacave", Beira Alta, XXXIX, 3 et 4, 1980, pp. 291-342, et XL, 1, 1981, pp. 10-11.

Correia 1972: CoRreia (V.). - "Arqueologia de Cárquere”, Obras, IV, Coimbra, 1972, pp. 261-263.

Dias 1985: DIAS (M. M. A.). - "Da latinização onomástica à romanização onomástica no processo da aculturação dos Igaeditani”, Symbolae Ludovico Mitxelena Septuagenario Oblatae, quas edidit José L. Melena, Vitoria, 1985, pp. 557-562.

Dias 1986: DIAS (M. M. A.). - "Inscrições romanas de Cárquere, Resende, na colecção epigráfica do Museu Nacional de Arqueologia e Etnologia", O Archeòlogo Português, série IV, 4, 1986, pp. 185-202.

Conimbriga, 35 (1996) 69-106 
Dias 1987: DiAs CM. M. A.). - "Antroponomia de Cárquere, Resende, Viseu (Lusitânia Portuguesa)", Actas del IV Coloquio sobre Linguas e Culturas Paleohispanisas (Victoria/Gasteiz, 6-10 Mayo 1989), Veleia, 2-3, 1987, pp. 195-203.

Dias 1989/92: DIAS (M. M. A.). - "Para um repertorio das inscrições romanas no territorio portugués”, Euphrosyne, XVII, 1989, pp. 373-384; XX, 1992, pp. 467-472.

Encarnação 1986: ENCARNAÇão (J.). - "Indigenismo e romanização na Lusitânia", Biblos, 62, 1986, pp. 451-464.

Encarnação 1994: ENCARNAÇão (J.). - "O monumento epigráfico romano, fonte para o estudo das migrações na Península Ibérica", Comunicação ao III Congresso Peninsular de História Antigua, em Vitória (à paraître dans Veleia).

Etienne et alii 1976: Etienne (R.), Fabre (G.), Le RouX (P.), Tranoy (A.). - Les dimensions sociales dans la romanisation de la Péninsule Ibérique des origines à la fin de VEmpire, Travaux du $\mathrm{VI}^{\mathrm{e}}$ Congrès International d'Etudes Classiques, Bucuresti-Paris, 1976.

Frankowski 1920: FrankowsKi (E.). - "Estelas discoideas de la Peninsula Iberica, junta para amplicacion de estudios et investigaciones sientificas.", Memoria, 25, 1920, p. 39, fig. 8, p. 151, fig. 66 .

Gonçalves da Costa 1977: GonçALVes DA Costa (A.). - História do bispado de Lamego, I, Lamego, 1977.

Gonçalves da Costa 1979: GonçALVes DA COSTA (A.). - História do bispado de Lamego, II, Lamego, 1979.

Hatt 1986: HATT (J. J.). - La tombe Gallo-romaine, Paris, 1986.

Jalhay 1951: JALHAY (E.). - "Lápides romanas da região de Cárquere, Resende", Broteria, LII, Lisboa, 1951, pp. 70-85.

Jesus 1947: JESUS (J. S.). - "Duas esteias funerárias lusitano-romanas na igreja de Almacave, Lamego", Biblos, XXIII, 2, 1947, pp. 545-550 et XXIII, 3, 1947, pp. 835-836.

Lambrino 1956: LAMBRINo (S.). - "Les inscriptions latines du musée Leite de Vasconcelos", O Archeòlogo Português, nova série, III, Lisboa, 1956, pp. 5-74.

Leite de Vasconcelos 1888: LeITE DE VAsconcelos (J.). - "Antiguidades de Cárquere", Revista Archeologica e Histórica, II, Lisboa, 1888, pp. 113-115.

Leite de Vasconcelos 1900: LeITE DE VASCONCELOS (J.). - "Antiguidades de Cárquere", O Archeòlogo Português, V, Lisboa, 1900, pp. 206-212.

Leite de Vasconcelos 1913: LeITE DE VASCONCELOS (J.). - Religiões da Lusitânia, II, 1905 et III, 1913.

Leite de Vasconcelos 1929: LeITE DE VAsConcelos (J.). - "Epigraphia do Museu Etnológico (Belém). Inscrições romanas", $O$ Archeòlogo Português, XXVIII, Lisboa, 1929, pp. 209-227.

Mantas 1984: Mantas (V. G.). - "A inscrição rupestre de estação luso-romana de Mogueira (Resende)", Revista de Guimarães, 1984, pp. 361-370.

Mattos 1948: MATTOS (A.). - "Inventário das inscrições do Douro Litoral", Douro Litoral, terceira série, I, Porto, 1948 , p. 65, n. $^{\circ} 81 ;$ p. 67, n. ${ }^{\circ} 83 ;$ p. 70 , n. ${ }^{\circ} 86$.

Reigota 1989: REIGOTA (J.). - "Estela a Valério Reburro, de Cárquere (Resende)", Licheiro Epigráfico, 32, 1989, n. ${ }^{\circ}$ 147; "Fragmento de estela funerária de Cárquere (Resende)”, Bicheiro Epigráfico, 32, 1989, n. ${ }^{\circ} 148$.

Conimbriga, 35 (1996) 69-104 
Sarmento 1887: SARMento (F. M.). - "Inscrições inéditas”, Revista de Guimarães, IV, 1887, p. 186-187.

Tovar 1977: Tovar (A.). - Les noms ibériques dans Vonomastique latine, Paris, 1977.

Tranoy 1981: Tranoy (A.). - La Galice romaine. Recherches sur le nord-ouest de la Péninsule Ibérique dans l'Antiquité, Paris, 1981.

Vaz 1982: VAZ (J. L. L). - "Breve catálogo das inscrições romanas de Lamego", Beira Alta, XLI, 1982, pp 507-512

Vaz 1983: VAZ (J. L. L). - "Lápide romana de Meijinhos", Beira Alta, 42(3), 1983, pp. 579-580.

Vaz 1986: VAZ (J. L. I.). - "Epigrafia romana de Cárquere - Mais cinco inscrições", Revista da Universidade de Aveiro/Letras 3, 1986, pp. 285-308.

Vives 1958: VIVES (J.). - "Características regionales de los formulários epigráficos romanos", Actas del Primer Congreso Español de Estúdios Clásicos, Madrid, 1958, pp. 485-492. 